\title{
Framework for Limit State Design of Geosynthetic-Reinforced Walls and Slopes
}

\author{
Dov Leshchinsky • Beongjoon Kang • Jie Han • \\ Hoe Ling
}

Accepted: 20 March 2014 / Published online: 3 April 2014

(C) Springer New York 2014

\begin{abstract}
Introduced is a unified limit state design framework for geosyntheticreinforced slopes and walls. It is demonstrated that limit state design is an essential step in design even though the usual perception is that the performance of such structures is "better than expected." A brief critical overview of commonly available analysis methods is presented. The typical design methodology which is an extension of conventional limit equilibrium (LE) analysis is discussed. It is shown that with the addition of the safety map tool, an optimized design can be achieved with relative ease. However, while the safety map can be used in design, the basic solution is still incomplete. Subsequently, the LE approach is generalized to include the complete solution, i.e., the required tensile force distribution along each layer considering reinforcement layout and anchorage properties. The modified approach produces a solution needed for stability including the required connection strength. It is based on free body equilibrium ensuring that at each point within the reinforced mass, the factor of safety is the same. That is, unlike the safety map which shows the corresponding safety factor at each location, the presented framework adjusts the required strength of the reinforcement so that the safety factor is the same constant everywhere. Limited parametric studies demonstrate the performance of the
\end{abstract}

D. Leshchinsky $(\bowtie)$

CEE Department, University of Delaware, Newark, DE 19716, USA

e-mail: dov@udel.edu

B. Kang

University of Delaware, Newark, DE 19716, USA

e-mail: bjkang@UDel.edu

J. Han

Department of Civil, Environmental, and Architectural Engineering,

The University of Kansas, Lawrence, KS 66045, USA

e-mail: jiehan@ku.edu

H. Ling

Department of Civil Engineering and Engineering Mechanics,

Columbia University, 500 West 120 Street, New York City, NY 10027, USA

e-mail: Ling@civil.columbia.edu 
new framework as well as that of the safety map approach. These studies show the impact of facing blocks, seismicity, backslope, quality of backfill, length of reinforcement, and effects of secondary short reinforcement. The results are compared with relevant experimental data. The agreement is reasonably good. Finally, a general link between the framework and actual design is briefly discussed.

Keywords Limit equilibrium - Limit state - Geosynthetics · Reinforced walls · Safety map $\cdot$ Slope stability

\section{Introduction}

Measured field data generally indicates that under typical conditions, the reinforcement force in geosynthetic-reinforced walls and slopes, termed as geosynthetic-reinforced soil (GRS), is mobilized less than predicted in design. Without adequate explanation, such an observation has led some to mistrust established design methodologies. In fact, based on forensic experience of the first author, a few designers and contractors had occasionally bypassed the standard of care assuming that current design is overly conservative. While reasonable conservatism is prudent, it seems that the main reason for defining a design as overly conservative is the lack of distinction between typical and atypical field conditions. Atypical or extreme conditions may exist during the lifespan of the structure, and these conditions must be addressed in any sound design. Such conditions should potentially consider situations such as heavy rainfalls resulting in increased degree of saturation of the backfill or vanishing toe resistance. Measured field data corresponding to an increasing degree of saturation is rare although numerous failures have occurred after heavy rainfall events.

Leshchinsky and Tatsuoka [24] pointed out that the apparent small mobilization of reinforcement force in GRS is mainly due to three reasons. One is the frictional strength of soil used in design which is significantly lower than the actual value when one considers a typical select backfill and its compaction level. For example, AASHTO $[1,2]$ limits $\phi$ to a maximum value of $40^{\circ}$ while allowing a default value of $34^{\circ}$. Consequently, while for the gradation and compaction required often $\phi$ could be more than $50^{\circ}$, in design, a typical value of $34^{\circ}$ is used. A second contributor to underestimation of reinforcement load is ignoring the toe restraint. Resistance generated by a 0.30 -m deep block can reduce the reinforcement load by as much as $50 \%$ (e.g. [25]). A third contributor is possible soil suction which generate an apparent cohesion in the backfill soil. Such apparent cohesion has large impact on stability rendering the reinforcement, in many cases, hardly needed. As an example, consider the failure shown in Fig. 1. It occurred simultaneously on both sides of the rounded corner. Forensic study indicated that layer by layer, sectors of reinforcement were not placed near the curved corner thus forming a triangular vertical prism of unreinforced soil in the upper tier where geogrids were missing. The obvious conclusion is that failure should have occurred as reinforced walls without reinforcement fail. However, the failure occurred about a year after the end of construction. Some vegetation on the face of the wall indicated that the backfill soil was moist. Increase in moisture increased the degree of saturation to a point where soil suction and subsequently apparent cohesion diminished to a level where lack of reinforcement led to failure. Adequate installation 

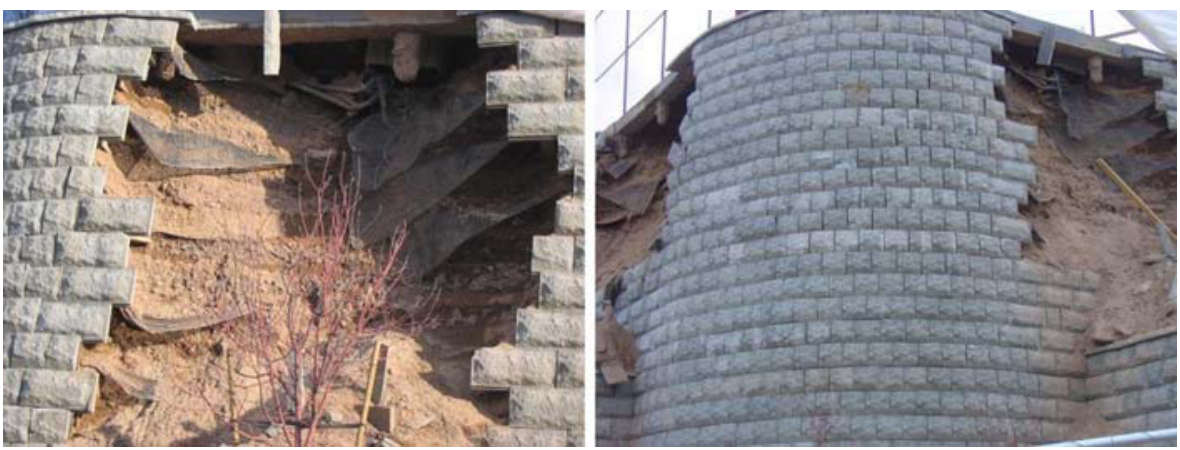

Fig. 1 Missing geogrids caused collapse one year after the end of construction: close up (left) and general view (right)

of geogrids in this case would have rendered the reinforcement is mostly dormant, i.e., hardly mobilized reinforcement. However, as the apparent cohesion was diminishing with an increased moisture, the geogrid strength would have been activated thus maintaining the stability of the GRS structure. Clearly, measuring the reinforcement load under normal conditions may lead to an unsafe conclusion regarding the level of conservatism of a design.

Sound geotechnical design needs to look at conditions which are likely to occur during the lifespan of the structure. These conditions could be considered "extreme" and should not include apparent cohesion or toe restraint. Under these conditions the structure must be in ultimate limit state (ULS) equilibrium. Limit equilibrium (LE) is one of several methods of analysis that can be used to assess limit state. This paper presents a framework for such analysis. It considers the layout of reinforcement in calculating the complete solution within the context of LE analysis. It yields the required tensile force along each layer thus rendering the connection and the maximum required strengths while considering the pullout capacity of each layer. It generalizes the work introduced by Han and Leshchinsky [10] to deal with any slope inclination considering backslope, various spacing, surcharge, and seismicity. Parametric studies show the impact of batter, length of reinforcement, secondary layers, soil frictional strength, and toe resistance.

The framework is an outgrowth of the method developed by Leshchinsky et al. [17] (see details at [16]), and later modified by Leshchinsky et al. [22]. Baker and Klein [4, 5] conducted a similar study for a zero batter wall; however, their methodology is different from the one presented here. In their analysis the force in upper layers was independent of layers below. Consequently, Baker and Klein [4, 5] predicted maximum load in the reinforcement that is larger than that produced by current design (e.g., by [1, 2]).

\section{Common Methods of Analysis}

The general objective of designing GRS is to determine the required strength and layout of the reinforcement so as to satisfy prescribed performance criteria. The layout and strength of the reinforcement are coupled, and therefore, there are many potential solutions yielding satisfactory performance. The selected solution should be economical, considering the cost of reinforcement, backfill, and construction. While such a 
consideration is at the discretion of the designer, this paper provides an analytical framework that may help one in making a rational decision. Following is a brief review of several design methodologies including empirical, semi-empirical, analytical, and complex numerical approaches:

i. Lateral Earth Pressure: Most design methods determine the reinforcement load based on calculated lateral earth pressures (e.g. [1, 2]). The approach is semiempirical (e.g., connection load is empirically selected). The main advantage of this approach is its simplicity. However, experience shows that it could lead to overly conservative selection of reinforcement strength. Equally important, it is limited to walls with batter up to $20^{\circ}$, to very simple geometry, to homogeneous backfill, and to a competent foundation. It has limited consideration of the interaction among reinforcement layers, and therefore, it offers little insight in terms of producing an optimized design which may include secondary layers or short primary reinforcement layers at some elevations. In fact, one may question the validity of the predicted lateral earth pressures irrespective of the reinforcement layout.

ii. K-Stiffness: This approach (e.g. [27]) is empirical, based on statistical correlations of field data obtained from various sources. This data has been collected under normal operating conditions, and therefore, the method is considered for "working load conditions." It implicitly includes unknown apparent cohesion and toe resistance that may have existed in the field during the data collection. Hence, it is not clear how one can configure the contribution of any of these significant factors when looking at limit state conditions. In fact, selecting geosynthetic based on the K-stiffness method may yield insufficient long-term strength needed to ensure ULS conditions. Similar to lateral earth pressures approach, the K-stiffness does not offer solution to cases with batter greater than $20^{\circ}$, it is limited to very simple geometry for which empirical calibrations were made, it offers little in terms of producing an optimized design which includes secondary layers or shorter reinforcement at some elevations, and it does not includes information such as where the maximum load in each reinforcement layer is located thus making calculations related to pullout resistance somewhat meaningless. Calibrating the K-stiffness to seismic loading or to water infiltration is also a limitation of the approach. It seems that the K-stiffness approach is a "black box" approach as it lacks any consideration of statics. Leshchinsky [14] has shown that this approach grossly violates global equilibrium under typical limit state conditions. It is considered unsafe since toe resistance (e.g. [25]) and apparent cohesion are not explicitly accounted for.

iii. Continuum Mechanics: This approach is numerically based (finite element (FE), Finite Difference (FD)). It is comprehensive in a sense that basic rules of mechanics are considered, accounting for boundary conditions and detailed material constitutive behavior. It is valid for slopes, walls, stratified soil, water, and more. To obtain reliable results at working load conditions (e.g., displacement), quality field data is needed, an unusual "commodity" in geotechnical practice. FE and FD results at a limit state could be reliable; however, it requires a designer with good understanding of possible numerical "traps." Furthermore, it would be extremely complicated to integrate this approach into the framework presented in this paper. 
iv. Limit Analysis (LA): Numerical upper bound in LA of plasticity yields kinematically admissible failure mechanisms; i.e., it is not necessary to assume arbitrarily a mechanism as done in LE which is an advantage when complex problems are considered. LA can deal with layered soil, complex geometries, water, seismicity, etc. It is valid for slopes and walls. LA can be integrated into the presented framework. However, because of limited familiarity of practicing engineers with LA, it may take a while before this valuable tool is accepted routinely in design.

v. Limit Equilibrium (LE): It can be applicable for complex problems including walls, slopes, complex geometries, layered strata, water, seismicity, etc. Its application to reinforced soil problems is merely an extension of an approach that has been used for decades in other geotechnical problems. One concern with LE (and LA) is its lack of direct consideration of "compatibility" between dissimilar materials. However, in unreinforced soil problems, consideration is given to the selection of material properties and prevailing failure mechanisms when vastly different soil layers exist. In fact, ULS of materials that are actually not very ductile is done routinely using LA, e.g., steel-reinforced concrete. A thirty-year experience shows that the use of LE in conjunction with soil and geosynthetics, both "ductile" materials, is not much of an issue. However, material properties for ULS design should be carefully selected.

LE is simple to apply. Also, there is a vast experience with the LE approach in design of critical geotechnical structures. If properly used, it may yield reasonably good agreement with experimental data (e.g., see later in this paper) as well as with results stemming from higher hierarchy in mechanics such as FE and FD (e.g. [20]). Current LE design of geosynthetic structures is concerned mainly with global instability. The framework presented in this paper extends its use to local conditions thus yielding the required reinforcement strength along each layer considering the multilayers layout, the geometry of the structure, the backfill types, and other relevant factors.

\section{Current $L E$ Modified Approach: the Safety Map Tool}

Some slope stability software packages offer a diagnostic tool that facilitates optimal design. This tool is called safety map, and it was formally introduced by Baker and Leshchinsky [6]. It is a color-coded map that, for a specific failure mechanism, shows the distribution of the safety factors within the soil mass thus indicating zones where the margin of safety is too low or where it is excessively high. The safety map in the context of reinforced soil indicates whether the specified strength and length of reinforcement produces adequate stability. The specified strength of reinforcement along its length is illustrated in Fig. 2. Note that at any location along the reinforcement, its strength is limited by either its intrinsic rupture strength or its pullout resistance, whichever value is smaller. Pullout resistance depends on the overburden pressure, reinforcement anchorage length, and reinforcement-soil interface properties. At its front side, pullout is superimposed on the connections strength when moving from the front. The pullout resistance shown in Fig. 2 varies linearly thus reflecting simple problem with zero batter and a horizontal crest. For more involved boundary conditions, the pullout resistance function will not be linear. 
Fig. 2 Available tensile resistance along reinforcement in current design

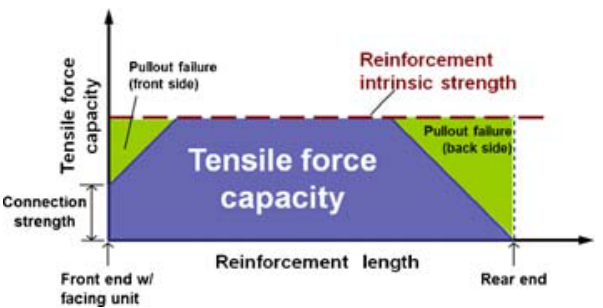

The factor of safety (Fs) used in current LE methods is related to the soil shear strength. It signifies the value by which the soil strength should be reduced to attain equilibrium at a limit state. The reciprocal value of Fs (1/Fs) signifies the average level of soil shear strength mobilization. The safety factors have similar meaning to Fs except that their value at any location is larger than Fs unless it is examined on the trace of the critical slip surface where it degenerates to Fs.

The usefulness of the safety map in the context of reinforced soil design using current LE is best demonstrated through an example problem. The following example problem, as well as other instructive examples, was originally published by Leshchinsky [18] with additional elaboration in [19].

Consider the problem of stability of multitiered slope/wall augmented by bedrock as detailed in Fig. 3. The design objective is to efficiently determine the required layout and strength of reinforcement to ensure sufficient margin of safety. The slope of the lower tier is $2(\mathrm{v}): 1(\mathrm{~h})$ while the top tier is at $20(\mathrm{v}): 1(\mathrm{~h})$. According to AASHTO's definition, this is a case of tiered walls over tiered slopes and as such, there are no clear design guidelines. Note that the foundation soil is comprised of a 2.5-m thick layer of residual soil possessing residual drained shear strength of $\phi=15^{\circ}$. Also note that the bedrock defines a slender structure while effectively limiting the depth of potential slip surfaces.

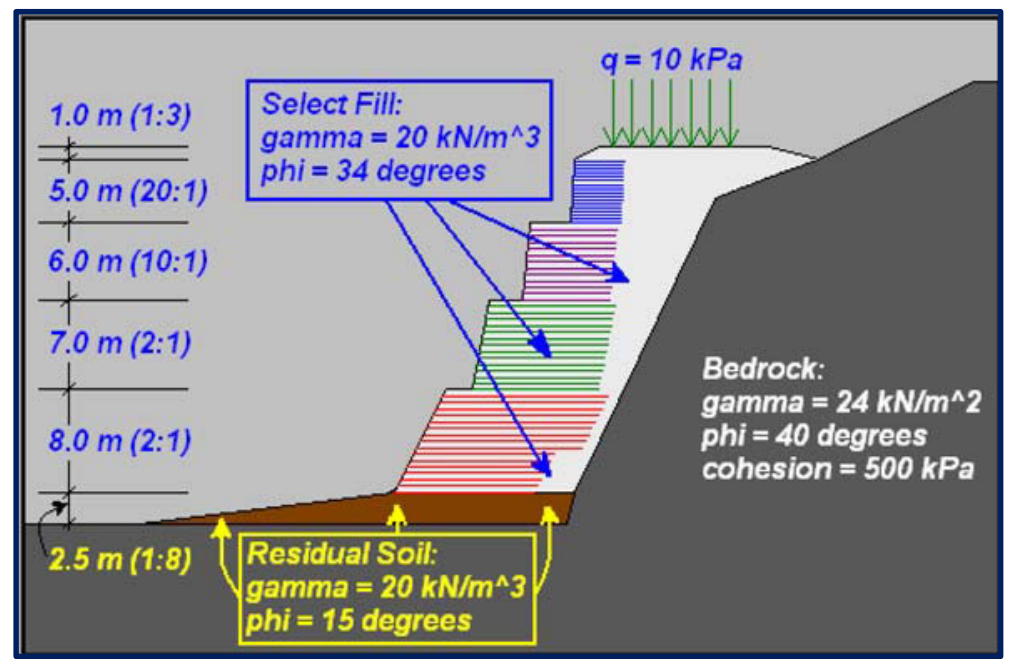

Fig. 3 The basic problem of multitiered slope/wall 
For granular unreinforced slopes, the critical slip surface always coincides with the steepest slope surface. The corresponding factor of safety for this problem then is trivial. Its value equals to $\tan (\phi) / \tan (\beta)$ where $\beta$ is the angle of the steepest slope which exists in the upper tier; $F s=\tan \left(34^{\circ}\right) / 20=0.034$. Figure 4 uses rotational failures combined with Bishop's analysis. It shows the location of the critical circle. By itself, this surface is of little value when designing for reinforcement. However, the red zone in the safety map shows that, practically, most of the granular backfill needs to be reinforced since the safety factor is less than 1.3 nearly everywhere. Note that for reinforced slopes, FHWA requires Fs $\geq 1.3$. The safety map, Fig. 4, indicates visually the zones within which the safety factor is unsatisfactory.

As a first iteration in the design process, the reinforcement layout shown in Fig. 3 is specified. The long-term design strength of the reinforcement for the bottom tier is $80 \mathrm{kN} / \mathrm{m}$; for the second tier, it is $50 \mathrm{kN} / \mathrm{m}$; for the third tier, it is $30 \mathrm{kN} / \mathrm{m}$; and for the top tier, it is $8 \mathrm{kN} / \mathrm{m}$. Rerunning the reinforced problem using Bishop's analysis yields the safety map shown in Fig. 5. The minimum factor of safety now is 1.29 (i.e., practically acceptable), and its corresponding critical circle is limited by the bedrock. The safety map implies the following:

1. The safety factor everywhere meets the required minimum of 1.3 . The map shows that for a rather large zone, the range of safety factors is between 1.3 and 1.5 (i.e., typical economical range for safety). Hence, the selected strength and length of the reinforcement is adequate to resist rotational failure economically.

2. The red zone extends into the residual soil and is restricted by the bedrock. Hence, although the red zone in Fig. 5 indicates an economical selection of reinforcement, it also signals different potential failure mechanisms that can adapt to the given geology producing a more critical situation.

Figure 6 shows the safety map employing two-part wedge translational failure mechanism combined with Spencer's stability analysis. Slip surfaces along the interface with the foundation, as well as along each reinforcement layer, are examined. The corresponding safety map implies the following:

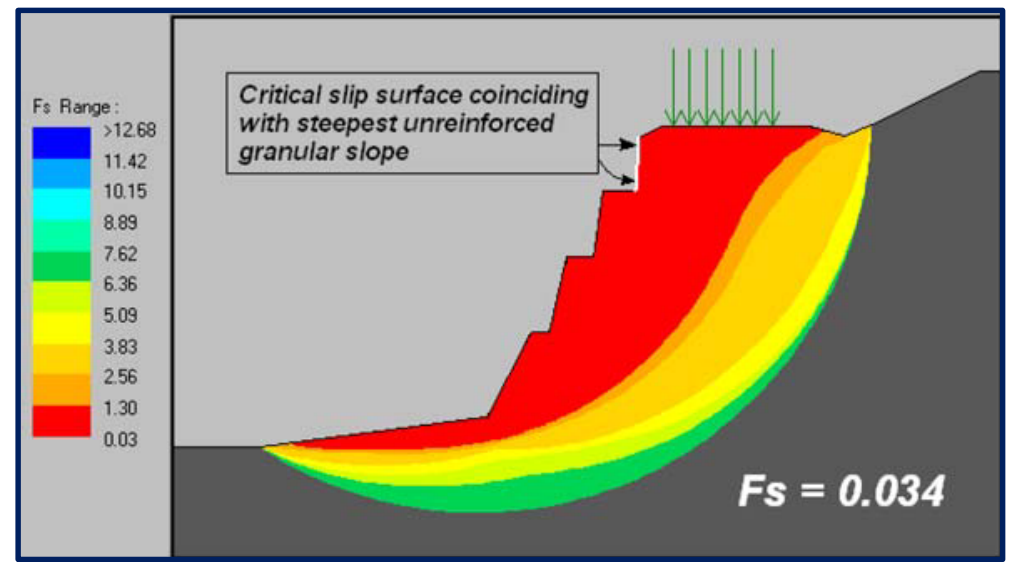

Fig. 4 Safety map for the unreinforced problem using Bishop's analysis 


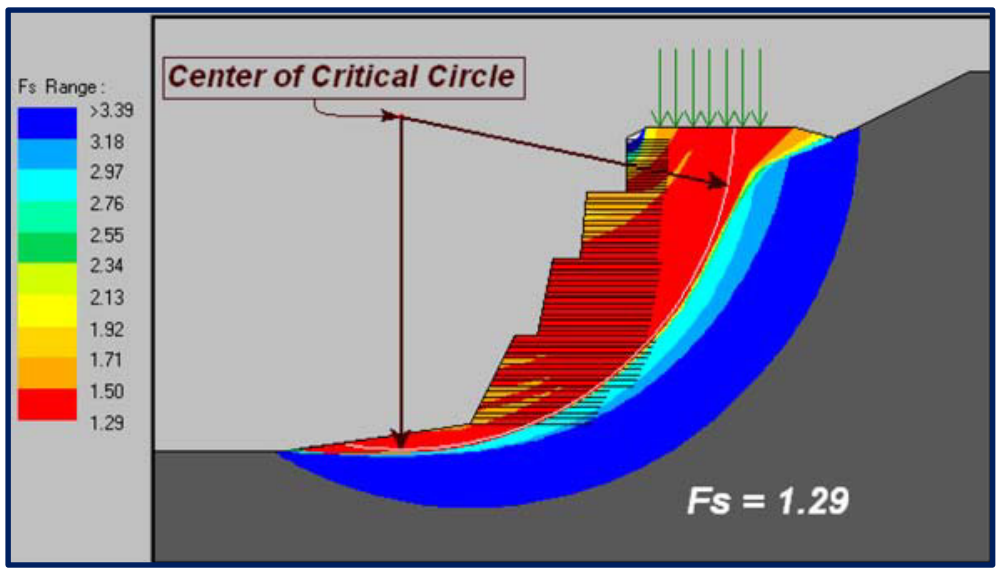

Fig. 5 Safety map for the reinforced problem using Bishop's analysis

1. The factor of safety for the initially assumed reinforcement is 0.9 , much lower than the acceptable value of 1.3. As can be seen, the critical slip surface propagates along the interface with the foundation (top of residual soil), extending beyond all reinforcement layers and limited by the bedrock.

2. The red zone signifies the range in which the safety factors are less than 1.3, i.e., unacceptable values. As seen, there are zones within each tier in which the safety factors are unacceptable as their values are still less than 1.3.

3. Clearly, the reinforcement for the top tier must be stronger. It also must be stronger for the tiers below as well.

4. While stronger reinforcement will improve stability against failures within the reinforced soil zones in all four tiers, it will not resolve the problem of failure around the reinforcement. Lengthening the reinforcement layers in the second and perhaps the third tier can solve this problem as reinforcement layers intersect the critical two-part slip surface.

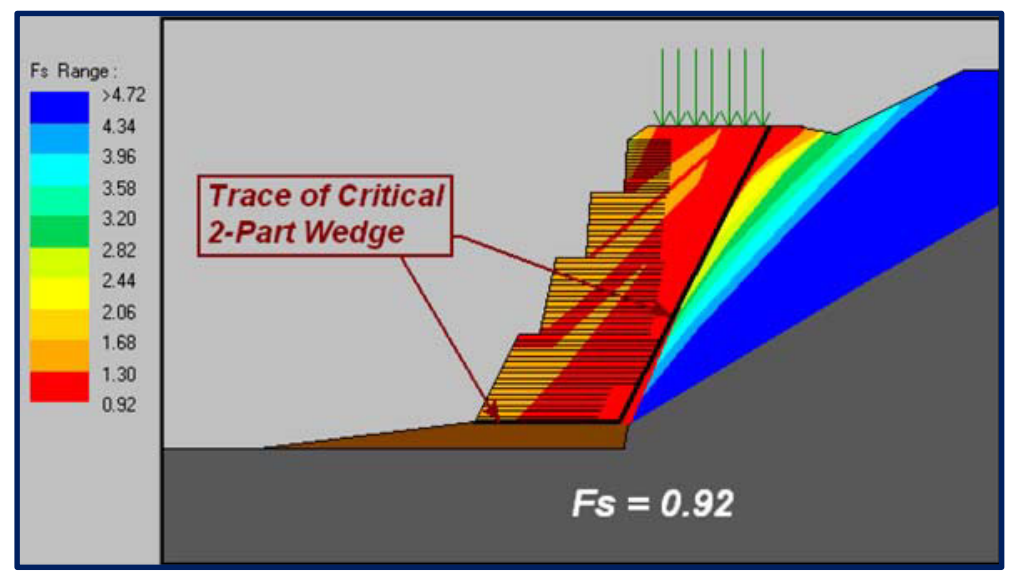

Fig. 6 Safety map for the reinforced problem using two-part wedge translational surfaces 
The lesson from using the two-part wedge translational mechanism combined with the initially assumed layout shows that one needs to increase both the strength and length of reinforcement. The depth of the red zone in the safety map suggests the extent to which the reinforcement should be lengthened; the existence of the red zone within the reinforced zone implies the need for increase in reinforcement strength. One can now lengthen and strengthen the reinforcement until the factor of safety is 1.3.

Figure 7 shows the safety map employing three-part wedge failure mechanism combined with Spencer's stability analysis. Translational failure mechanisms within the problematic zone, the foundation soil, are examined. The safety map implies that:

1. The factor of safety for the initially assumed reinforcement is 0.7 , much lower than the permissible value of 1.3. As can be seen, the critical slip surface propagates within the foundation, the residual soil, extending beyond all reinforcement layers and limited by the bedrock.

2. The red zone signifies the range in which the safety factors are less than 1.3, i.e., unacceptable values. As seen, there is one such zone extending between the rear of the reinforcement and the bedrock as well as within the entire foundation soil zone.

3. The safety map implies that while increasing the strength of the reinforcement may narrow the red zone, it is not likely to eliminate it altogether. Lengthening of the reinforcement in the three upper tiers may help but not likely to render a safe and economical design.

4. The safety map indicates that the residual soil creates a zone which decreases stability significantly. A logical effective solution in this case could involve ground improvement such as replacement of the residual soil before construction of the tiered system starts. This will also increase the resistance to direct sliding failure depicted by the critical two-part wedge in Fig. 6.

The safety map corresponding to the three-part wedge mechanism implies that replacing the residual soil may produce a satisfactory solution. Rerunning the problem with the foundation soil the same as the reinforced one (i.e., $\phi=34^{\circ}$ ) yields a factor of safety of 1.26 (as compared to 1.3) for the three-part wedge, a nearly acceptable value.

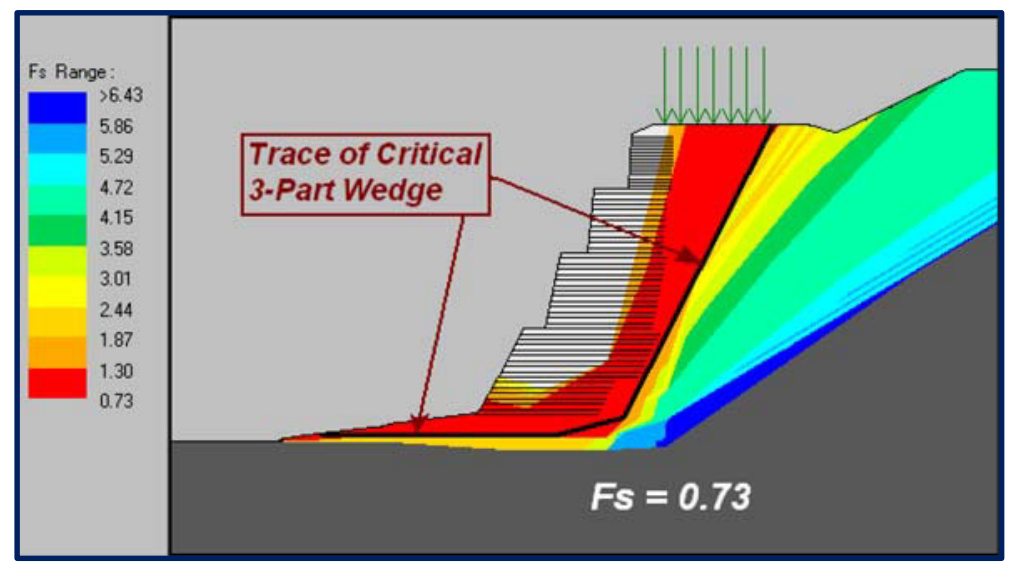

Fig. 7 Safety map for the reinforced problem using three-part wedge surfaces 
The safety map shown in Fig. 8 is a result of rerunning the problem for the two-part wedge translational mechanism. As seen, the problem associated with the foundation soil is resolved also for the two-part wedge (in fact, the safety factor along the foundation now is 1.37). The red zones in which safety factors less than 1.3 are within the second, third, and fourth tiers. These zones indicate that only a slight increase in reinforcement strength is needed; the length is adequate. Such an increase in reinforcement strength in the three upper tiers is likely to produce a rather economical utilization of the reinforcement as the range of the safety factors will be mainly between 1.3 and 1.5.

\section{Ideal Design: the General Framework}

The ideal design in this paper is an extension of the general framework presented by Han and Leshchinsky [10]. It is simpler, although similar to that presented by Baker and Klein $[4,5]$, and more importantly, it leads entirely different results which are with smaller required strength and length of reinforcement when identical problems are analyzed.

The general framework can be stated as the safety map in reverse. That is the required strength of the reinforcement is adjusted locally so as to mobilize the design shear strength of the soil equally anywhere within the reinforced soil mass. Constant factor of safety at any point within the mass means that potential failure surfaces have the same theoretical likelihood of passing through any point. Following the procedure used in MSE walls, one has to select a design value of the soil shear strength, $\phi$. For this value of $\phi$, the required strength of each layer along its length, $T_{\text {req } i}(x)$, is sought so as to produce at any point $F_{s}=1.0$. Since this work is design-oriented, possible contribution by cohesion is ignored. However, inclusion of true cohesion can be easily implemented in the analysis.

\section{Exceptions}

The objective of the free body analysis is to determine the value of $T_{\text {req }}(x)$ intersecting the test body to produce a system that is at the verge of failure (i.e., Fs=1.0). Actual

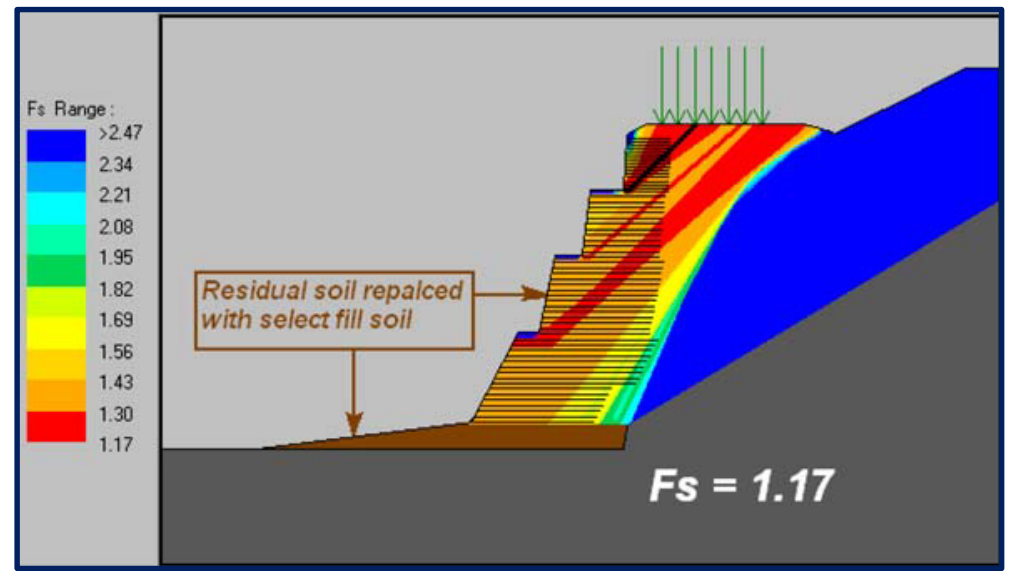

Fig. 8 Effects of replacing the foundation soil with select fill (two-part wedge) 
safety is achieved by specifying strength of reinforcement that is in excess of $T_{\text {req }} \mathrm{i}(x)$. However, under the following conditions, this objective may not be achievable:

i. When many layers are too short, insufficient pullout resistance may make it impossible to achieve an Fs=1.0, regardless of reinforcement strength. Simply, not enough tensile strength of reinforcement can be mobilized along some test bodies.

ii. When long reinforcement is used, the strength at the rear end of some layers, especially lower layers, may not be needed for stability. Only when the required tensile strength is "negative," Fs=1.0 is feasible. Of course, negative tensile resistance is a physical impossibility. The implication is that in zones where the reinforcement is not needed, achieving the goal of the analysis is not possible.

Viewed differently, the two exceptions imply that the framework looks for an optimal solution where the reinforcement is neither too short nor is it excessively long. The layout of all reinforcement layers, including its pullout resistance at each location, is considered in the process of rendering the optimal design.

\section{Selected Mechanism}

To demonstrate the framework in the context of LE analysis, baseline solutions were produced to enable parametric studies. To achieve that, a log spiral slip surface was selected in examining free bodies in an LE state. Such mechanism has experimental justifications (e.g., see Comparisons, [7, 21]) as well as theoretical justifications (e.g., kinematical admissible mechanism in upper bound of plasticity). Equally important, while LE analysis is usually associated with indeterminate statics, the log spiral produces a statically determinate solution. Assembling the moment equilibrium equation about the pole of the log spiral can be done rigorously, without resorting to assumptions in statics. The only unknown for such an equation is $T_{\text {req i }}(x)$ at its intersection with the trace of the log spiral. Hence, for a test or free body defined by a log spiral, the required tensile resistance in the reinforcement layers needed to generate $\mathrm{Fs}=1.0$ can be determined (see some explanation later).

The trace of the log spiral can be expressed in polar coordinates by $(r, \beta)$, and it is written as:

$$
r=A \exp (-\beta \psi)
$$

where $A=$ constant, $\beta=$ angle between a vertical line and the radius vector $r$, and $\psi=$ $\tan (\phi)$. Three parameters are needed to define a log spiral in a Cartesian coordinate system, its pole (Xc, Yc) and the constant $A$. In essence, it is similar to defining a circle using its center (Xc, Yc) and radius, $R$. However, it is more efficient to use different dependent parameters to define the log spiral, the angles of the tangents to the log spiral at its start and end points and its intersecting point at its emerging point —see Fig. 9. The relationships between the various parameters and the actual trace of the log spiral are related to basic trigonometry and were detailed by [15] as well as [12].

It is noted that a generalized rigorous stability method, employing more complex failure surfaces, can be used. 
Fig. 9 Defined trace of $\log$ spiral by $\theta 1, \theta 2$, and coordinate of emerging point

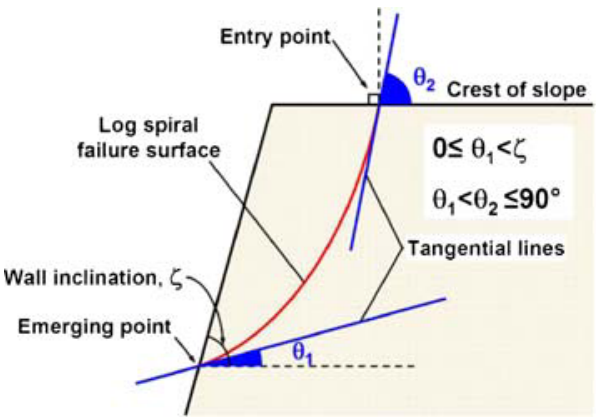

Basic Concept: Rupture and Pullout

To determine $T_{\text {req i }}(x)$, each reinforcement layer is discretized into small segments. Each tested log spiral passing through a particular segment will require $T_{\text {req i }}(x)$ which is determined by solving the moment equilibrium at an LE state. The log spiral requiring the maximum value of $T_{\text {req }}(x)$ at each reinforcement intersection, explained in the next section, renders the prevailing value of required tensile resistance at that location. While LE equilibrium requires a certain value of $T_{\text {req i }}(x)$, the capacity of some layers could be limited by its pullout resistance. In such a case, reinforcement layers below and possibly above should compensate for the lesser load carried by that layer. While this compensation may work for insufficient pullout resistance at the rear end of the reinforcement, at the front end, pullout resistance can be increased by adjusting the connection strength $T_{\mathrm{o}_{\mathrm{i}}}$. Figure 10 illustrates the synergy between the available pullout resistance and $T_{\text {req }}{ }_{\mathrm{i}}(x)$ along a layer. It shows that the value of $T_{\mathrm{O}_{\mathrm{i}}}$ is determined by translating the front end pullout resistance curve, parallel to itself, up to a point where the envelop is tangent to $T_{\text {req }} \mathrm{i}(x)$. The amount of translation at $x=0$ is the required connection strength, $T_{\mathrm{o}_{-} \mathrm{i}} \cdot T_{\text {req } \mathrm{i}}(x)$ calculated by the log spiral for LE state now can be mobilized up to the point where the rear pullout controls the tension capacity.

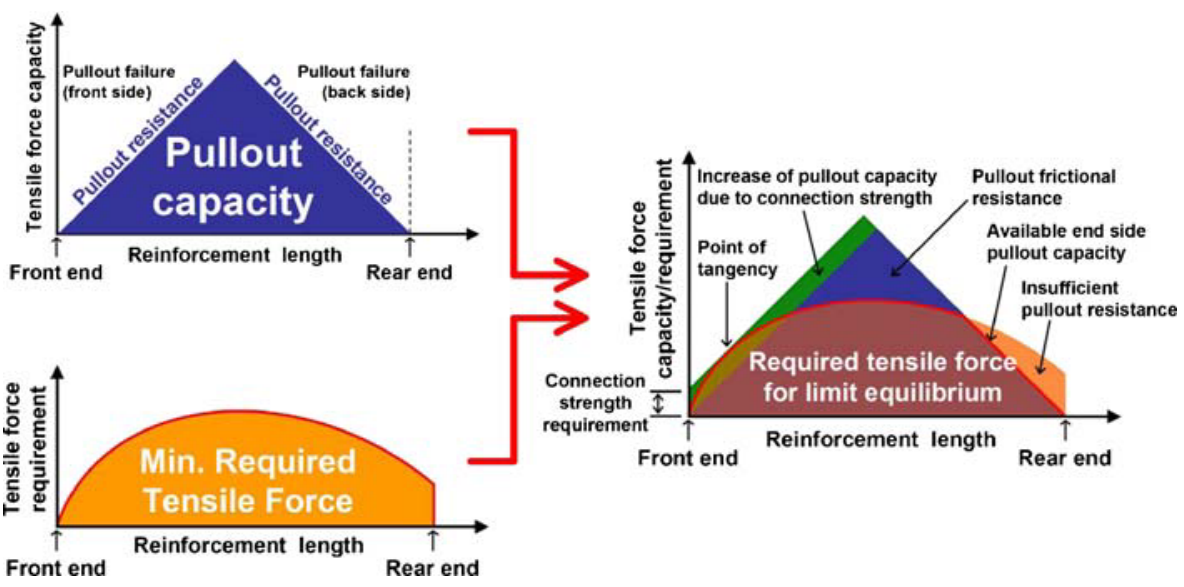

Fig. 10 Required reinforcement force augmented by front and rear pullout resistance 
The synergy of pullout and required tensile resistances shown in Fig. 10 implies that each reinforcement layer could be either too short or too long or have an ideal length. Figure 11 illustrates these three possibilities. If the reinforcement is too short, there will be insufficient pullout resistance to enable the development of $T_{\text {req }}$ i $(x)$ at its rear and therefore other layers will have to make up for this deficit in available tensile resistance which is needed for stability. If the reinforcement is too long, there is excessive pullout resistance thus possibly rendering dormant some of the reinforcement. If the length is ideal, the pullout resistance at the rear exactly enables the development of $T_{\text {req }}(x)$. In such a case, the rear end pullout resistance curve is ideally tangent to $T_{\text {req } \_} \mathrm{i}(x)$. Note that imposing pullout capacity which enables $T_{\text {req i }}(x)$ to develop necessarily creates a zone in which the reinforcement load capacity is somewhat excessive. In the ideal length case, this excess or redundancy is at both front and rear zone areas-Fig. 11.

\section{Top-Down Procedure}

The numerical procedure to determine $T_{\text {req } \mathrm{i}}(x)$ considering the rear end pullout is best illustrated by an example problem. Note that the minimum required connection resistance, $T_{\mathrm{o}_{-} \mathrm{i}}$, is determined only at the end of the iterative computational procedure. Mathematically, $T_{\mathrm{O}_{\mathrm{i}}}$ is an auxiliary parameter that must enable the development of $T_{\text {req } \mathrm{i}}(x)$. It is noted that the process leads to the required reinforcement and connection strengths and is termed baseline solution. Equipped with this information, the designer can assess an actual problem ensuring that it produces sufficient margins of safety.

Refer to Fig. 12. For a given geometry and layout, select an emerging point for log spirals below the top layer denoted reinforcement 1 . Initially, the emerging point is selected above or at reinforcement 2. Each test body defined by a log spiral is

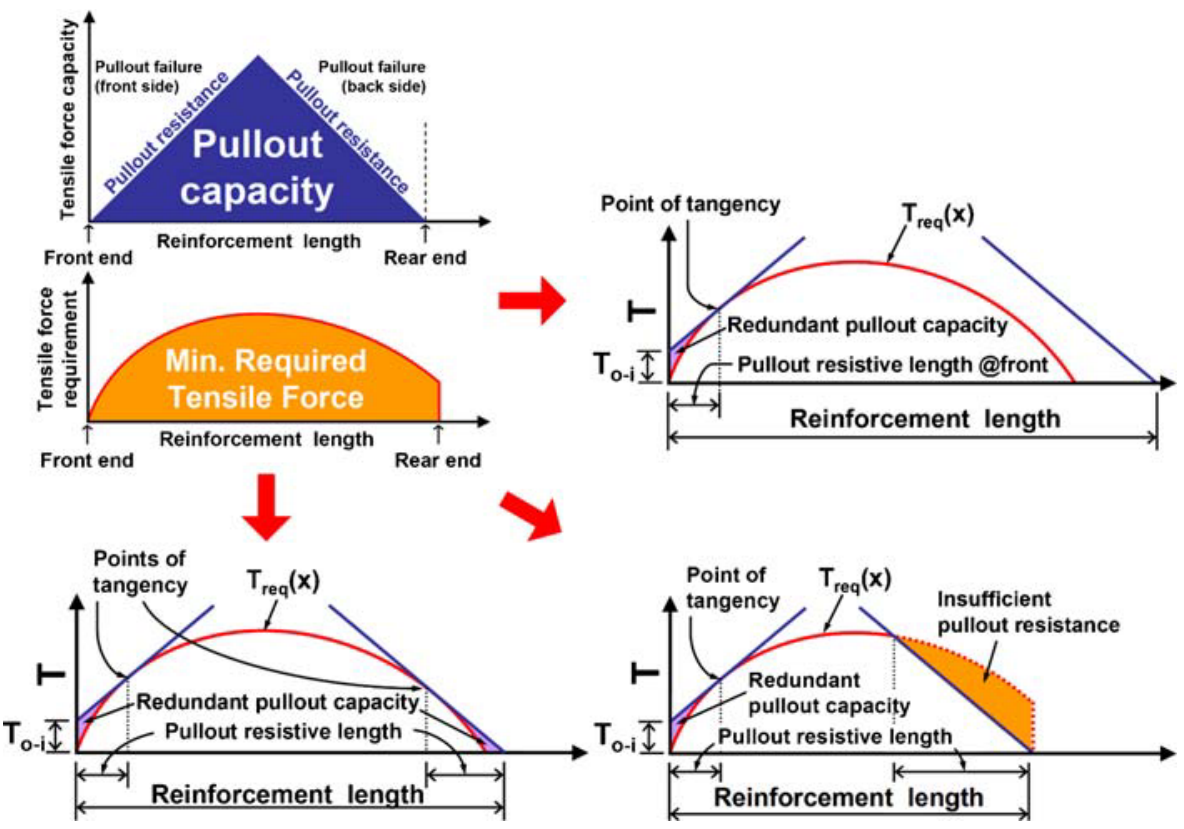

Fig. 11 Length of reinforcement layer i. Ideal (lower left), excessive (upper right), and short (lower right) 

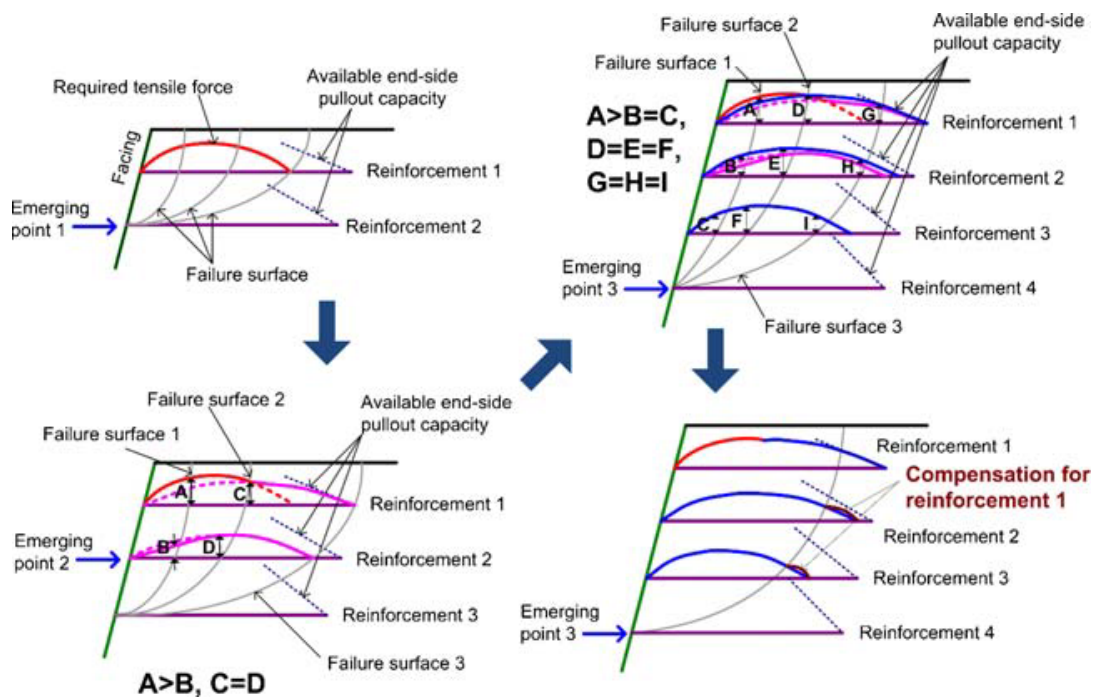

Fig. 12 Example of top-down procedure

considered at an LE state. The only unknown in the moment equilibrium equation for that body is the required tensile resistance of reinforcement 1 at its intersection with the analyzed log spiral. Many log spirals passing at the same point (or same segment that is approximated as a point) are considered by changing the other two parameters defining the log spiral, i.e., tangent angles at its entry and exit. The prevailing value at each point is the maximum required tensile resistance; i.e., it is a result of a numerical maximization process. As seen in Fig. 12, a curve of $T_{\text {req }}(x)$ is obtained; its value ensures that failure passing through any point along reinforcement 1 is equally likely to happen, i.e., all have the same factor of safety. Also note that the pullout capacity of the top layer at this stage is excessive thus enabling the full development of $T_{\text {req }} \mathrm{i}(x)$.

Next, select emerging point 2 , below reinforcement 2 and above or at 3 . The process to establish $T_{\text {req }} \mathrm{i}(x)$ is repeated; however, this time, it is for reinforcement 1 and 2. Log spirals intersecting two layers of reinforcement are initially assumed to mobilize equal strength from each layer unless that required strength is limited by insufficient pullout resistance capacity. However, if the evenly distributed required strength (dotted line in Fig. 12) at a point is less than the value required for previous emerging points (value $A$ in Fig. 12), the higher value prevails as it is required for an LE state for a log spiral emerging at a previous point. Subsequently, selecting for the two layers an equal strength value of $A$ means excessive strength for reinforcement 2. Hence, while the value $A$ is kept for reinforcement 1 , a reduced value of $B$ is assigned to reinforcement 2 . For the specific log spiral used to determine $B$ ("failure surface 1"), a state of LE exists as reinforcement 1 carries $A>B$. As seen in Fig. 12, for a certain log spiral ("failure surface 2"), the required resistance from the two layers is equal to each other, $C=D$. To the right of $C$, the required strength of reinforcement 1 is increased from its calculated value for the previous emerging point, to be equal to that of reinforcement 2 rendering LE state for all test bodies. Figure 12 indicates that rear end pullout of the top layer is nearly exceeded ("failure surface 3"); in fact, at this stage, reinforcement 1 has an "ideal" length. 

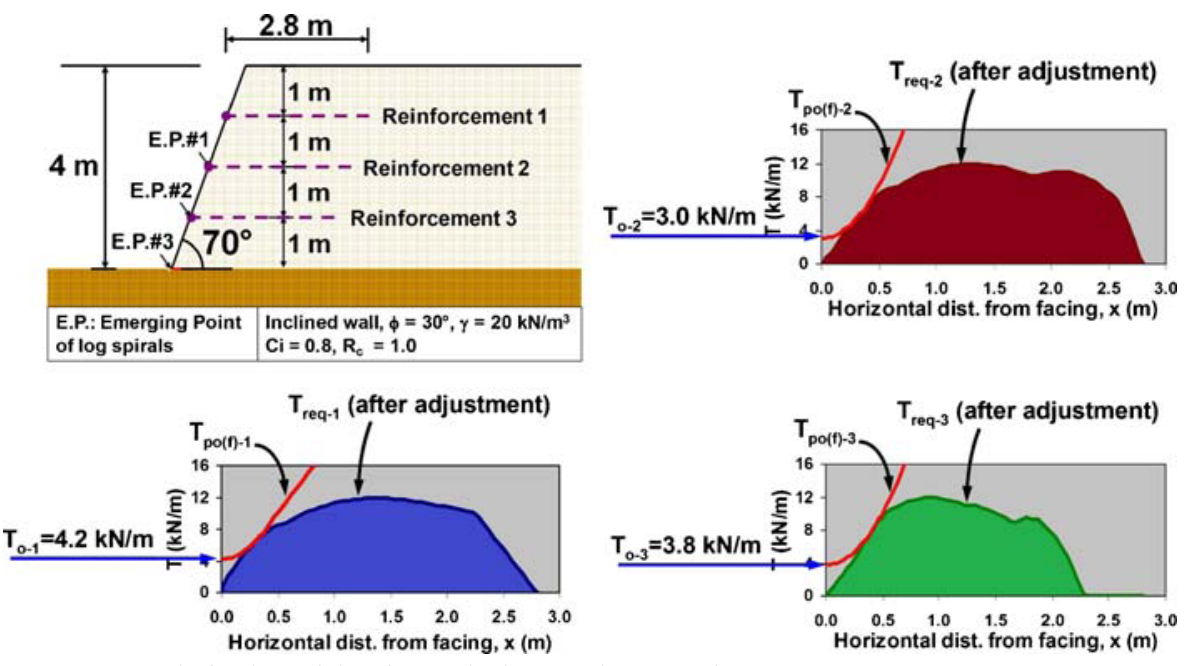

Fig. 13 Example for determining the required connection strength

The process is repeated at the third emerging point. In Fig. 12, for failure surface 1 the required tensile resistance in reinforcement 3 is the same as in 2 but smaller than in 1 (i.e., $A>B=C$ ). For failure surface 2, all layers require equal resistance, i.e., $D=E=F$. While failure surface 3 also renders equal load $G=H=I$, deeper surfaces require load contribution by reinforcement 1 that is larger than its pullout resistance enables. Consequently, in zones to the right of failure surface 3, the load in reinforcement 2 and 3 is increased to ensure an LE state by compensating for insufficient pullout resistance of the top layer.

The top-down process continues down to the toe elevation, generating $T_{\text {req }} \mathrm{i}(x)$ for all layers considering its rear pullout capacity. However, the required connection strength, $T_{\mathrm{o}_{\mathrm{i}}}$, enabling $T_{\text {req } \mathrm{i}}(x)$ at the front end has not yet been determined. Refer to the illustrative example in Fig. 13. It shows the results for a batter of $20^{\circ}, \gamma=20 \mathrm{kN} / \mathrm{m}^{3}$, and $\phi=30^{\circ}$. For this example, the required $T_{\text {req }}(x)$ was calculated using the top-down process. Pullout resistance is considered a function of overburden pressure and the interaction coefficient $\mathrm{Ci}$ (in this case $\mathrm{Ci}=0.8$ ). For batter greater than zero, the slope angle will affect the overburden pressure near the connection. As a result, the front end pullout resistance is not linear-Fig. 13. In this problem, the overburden at the rear end is uniform and there, the pullout varies linearly with its distance from the end of the

Fig. 14 Elements considered in the parametric studies

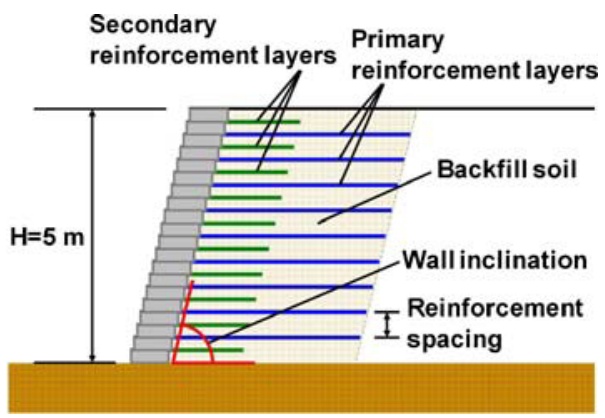


reinforcement. As can be seen, the front end pullout nonlinear resistance curves were translated (i.e., copied parallel to themselves) until being tangent to $T_{\text {req } \mathrm{i}}(x)$. Such translation renders $T_{\mathrm{O}_{\mathrm{i}}}$ for each of the layers. It is noted that the point of tangency could be sensitive to numerical inaccuracy. However, the resulted $T_{\mathrm{o}_{-} \mathrm{i}}$ values are small thus not posing a practical problem.

If the reinforcement is equally spaced and sufficiently long while the connection is sufficiently strong, the top-down limit state approach will result in equal mobilization of maximum tensile resistance in each layer, $T_{\max -\mathrm{i}}$. Such a phenomenon at the verge of collapse is implied by some experimental work [28]. This is also being used in geotechnical practice of reinforced slope stability analysis. It is noted that upon minor modifications, other empirically based distributions [25] can be implemented in the presented framework.

Fig. 15 Baseline solution for $\zeta=$ $90^{\circ}, \phi=30^{\circ}, L / H=0.7, S_{\mathrm{v}}=0.5 \mathrm{~m}$, $R_{\mathrm{c}}=1.0$, and $\mathrm{Ci}=0.8$
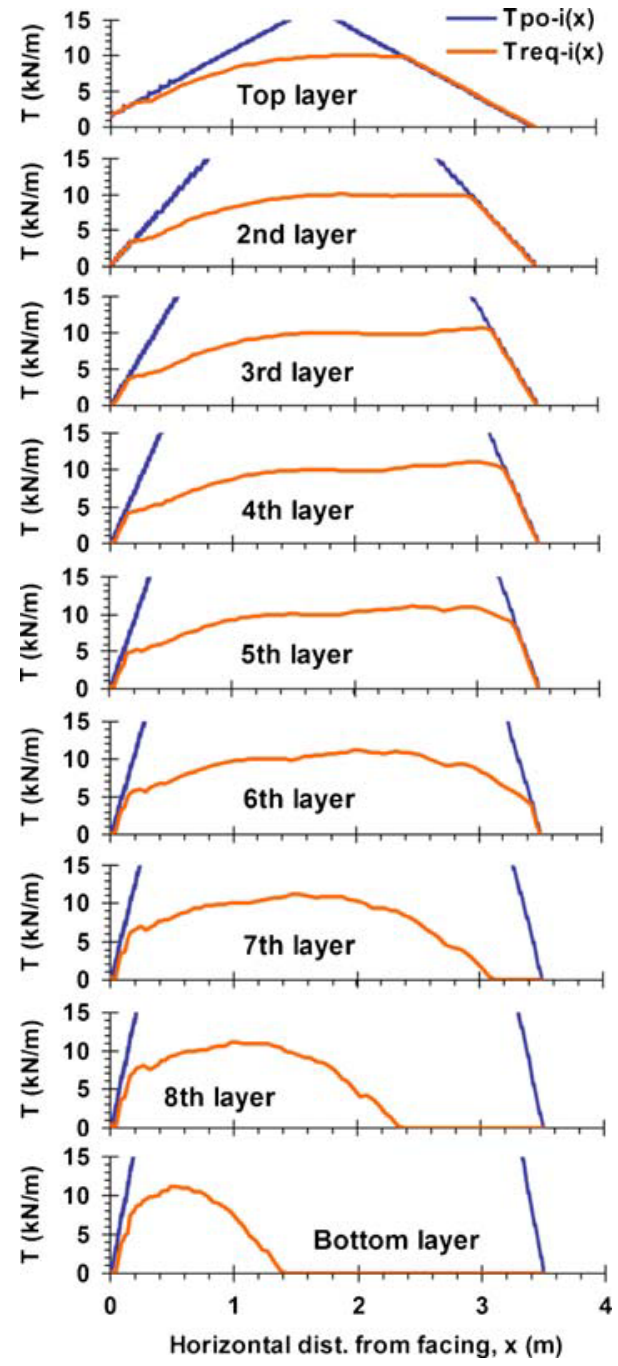
Fig. 16 Baseline Solution for Considering Frictional Strength of $20^{\circ}$ to $50^{\circ}$
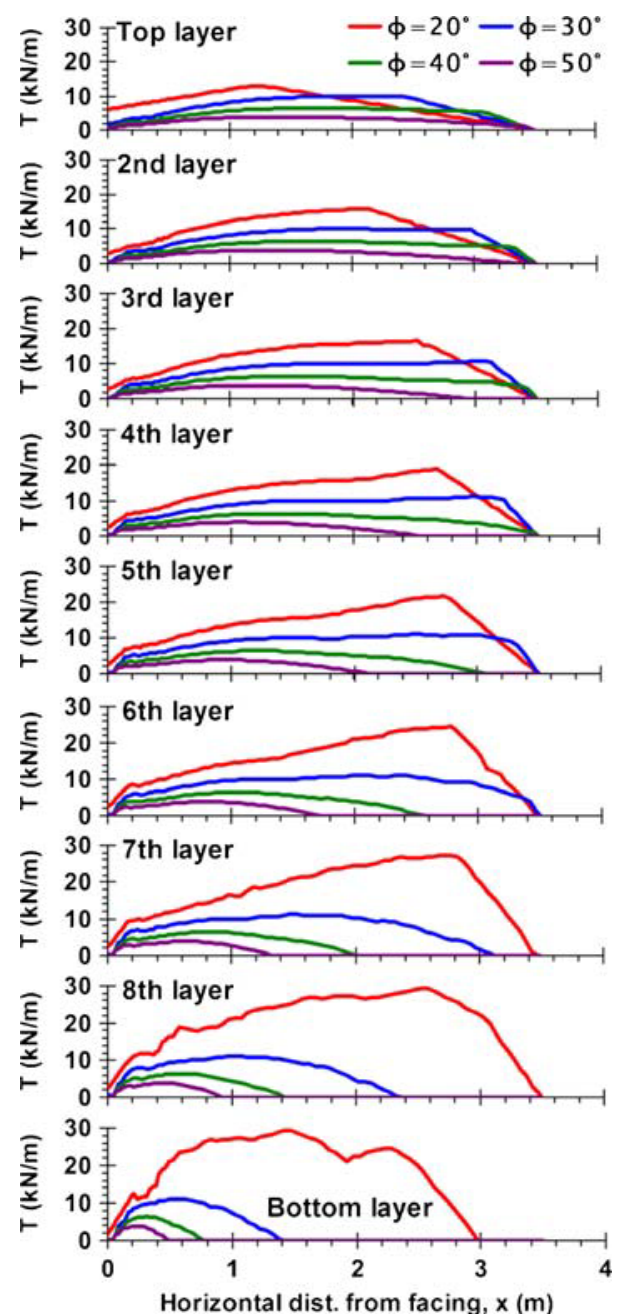

a

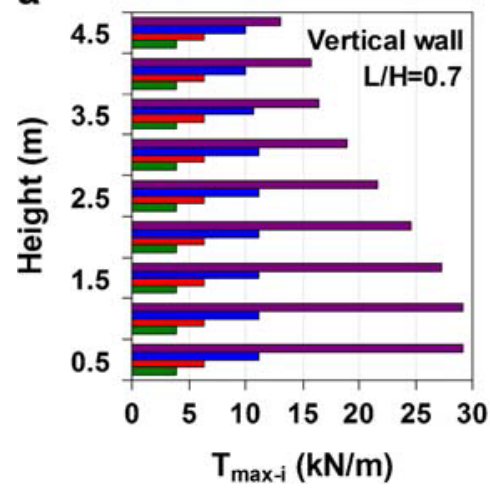

b

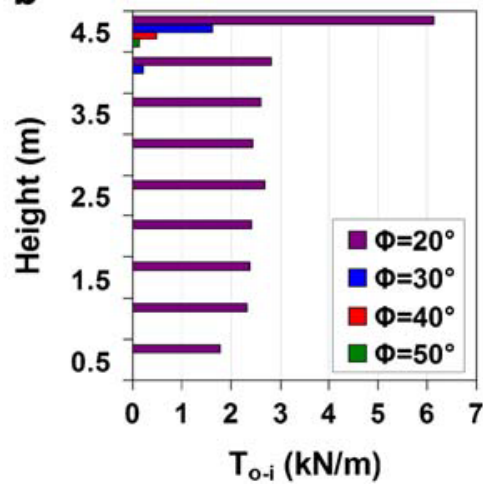

Fig. 17 Effects of $\phi$. a $T_{\max -\mathrm{i}}$, and $\mathbf{b} T_{\mathrm{o}-\mathrm{i}}$ 


\section{Parametric Studies}

Figure 14 shows the scope of problems for which baseline solutions were generated forming comprehensive parametric studies. Not indicated in Fig. 14 are the studies of pseudostatic acceleration, changes in coverage ratio, backsloped crest, and facing effects. In all cases, unit weight of soil was $\gamma=20 \mathrm{kN} / \mathrm{m}^{3}$.

Note that the following notation is used: $\zeta=$ wall/slope inclination, $H=$ height of wall/slope, $L=$ length of primary reinforcement, $S_{\mathrm{v}}=$ vertical spacing of reinforcement, $R_{\mathrm{c}}=$ coverage ratio, and $\mathrm{Ci}=$ interaction coefficient.

Figure 15 shows the computed $T_{\text {req } \mathrm{i}}(x)$ and $T_{\mathrm{O}_{-} \mathrm{i}}$ for vertical wall with granular backfill having $\phi=30^{\circ}$. For select backfill, $\phi=30^{\circ}$ could be considered as low (e.g., AASHTO allows for default value of $34^{\circ}$ when its select backfill is specified); however, it is a traditional "benchmark" number in geotechnical practice thus worthy of consideration. It is seen that rear end pullout resistance of the upper 6 layers is mobilized. The length of reinforcement is "excessive" only for the lower 3 layers. The top layer requires some connection strength to enable the development of tensile resistance along the reinforcement; for other layers, the required value is negligibly small.

Figure 16 shows the impact of soil strength. It is noted that soil with $\phi=20^{\circ}$ is unlikely to be used. However, there are two reasons for its inclusion in the parametric study. First, if Fs definition in conventional slope stability is used (say, Fs $=1.5$ ) than $20^{\circ}$ represents soil with actual strength of about $29^{\circ}\left\{=\operatorname{atan}\left[\mathrm{Fs} \tan \left(20^{\circ}\right)\right]\right\}$. Second, it is instructive as it demonstrates the required tradeoff for using truly low-quality backfill. Figure 16 indicates dramatic nonlinear increase in required strength of reinforcement as $\phi$ decreases. Connection strength goes up too. For high $\phi$, many layers are longer than needed. However, for low $\phi$, nearly all layers having $L / H=0.7$ are "short"; thus, their contribution is limited by their pullout resistance capacity.

In design, the maximum required tension load in each layer, $T_{\max -\mathrm{i}}$, and connection load, $T_{\mathrm{o}-\mathrm{i}}$, are sought. Figure 17 provides these values as extracted from the computations generating Fig. 16. Figure 17 a clearly shows that as $\phi$ decreases, $T_{\text {max-i }}$ becomes non-uniform implying inefficient use of reinforcement. It can be verified that $\max \left(T_{\max -i}\right)$ calculated here is about half of the value produced by AASHTO's design. As an example, for $\phi=30^{\circ}$, Fig. 17 a indicates that $\max \left(T_{\max -\mathrm{i}}\right) \approx 11 \mathrm{kN} / \mathrm{m}$ whereas AASHTO will yield for the bottom layer, which has $S_{\mathrm{v}}=0.75 \mathrm{~m}, \max \left(T_{\max -\mathrm{i}}\right) \approx 23 \mathrm{kN} / \mathrm{m}$. Similarly, for $\phi=40^{\circ}$,

a

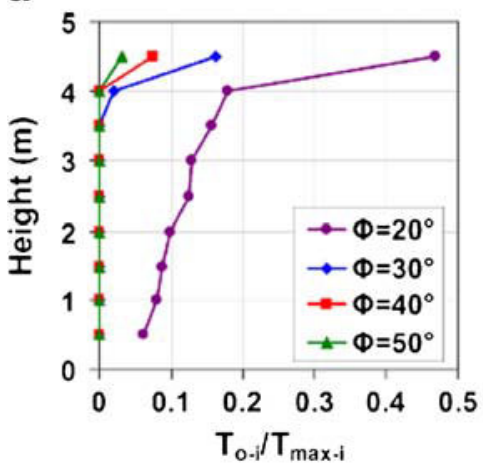

b

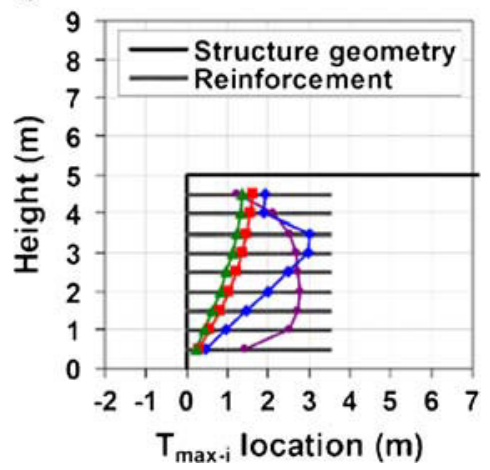

Fig. 18 Effects of $\phi$. a $T_{\mathrm{o}-\mathrm{i}} / T_{\max -\mathrm{i}}$ and $\mathbf{b}$ locus of $T_{\max -\mathrm{i}}$ 
a

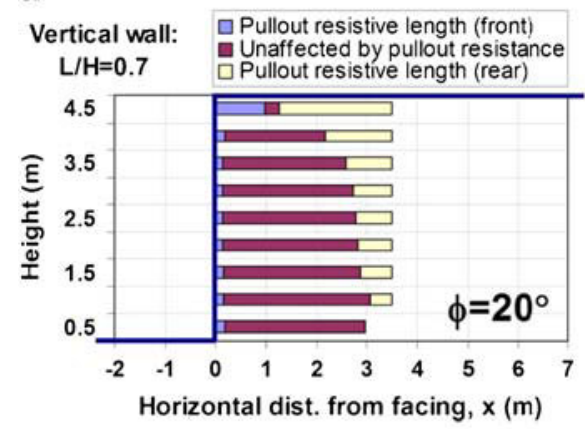

C

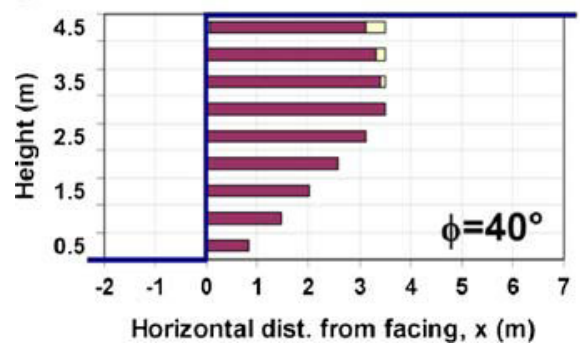

b

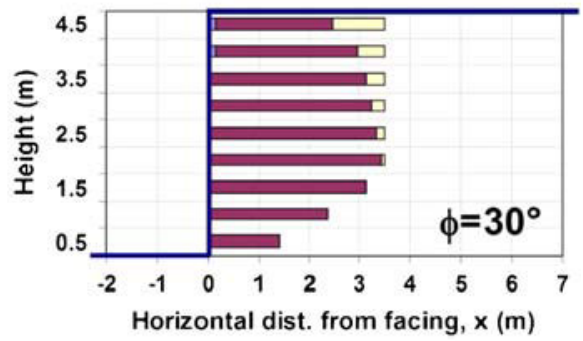

d

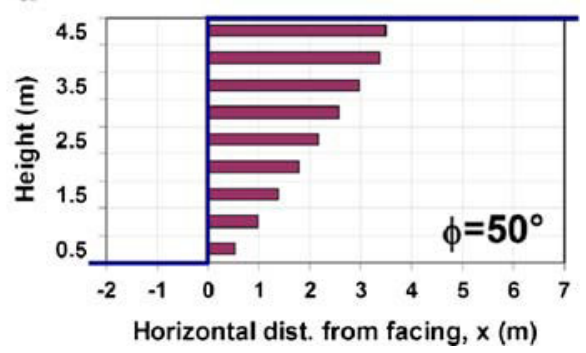

Fig. 19 Pullout resistive lengths. a $\phi=20^{\circ}, \mathbf{b} \phi=30^{\circ}, \mathbf{c} \phi=40^{\circ}$, and $\mathbf{d} \phi=50^{\circ}$

$\max \left(T_{\text {max-i }}\right) \approx 6 \mathrm{kN} / \mathrm{m}$ whereas AASHTO yields $\max \left(T_{\text {max }-\mathrm{i}}\right) \approx 15 \mathrm{kN} / \mathrm{m}$. This outcome is significant as $\max \left(T_{\max -\mathrm{i}}\right)$ determines the required long-term strength of reinforcement. The connection load, $T_{\mathrm{o}-\mathrm{i}}$, seen in Fig. $17 \mathrm{~b}$ goes up as $\phi$ goes down.

Figure 18a shows that the connection load is a small fraction of the required maximum tensile resistance $T_{\max -\mathrm{i}}$. However, this value can be as much as half of $T_{\max -\mathrm{i}}$ for low $\phi$ values. For vertical reinforced slope, the main relative impact is in upper layers. In this conjunction, it is interesting to note that AASHTO requires that $T_{\mathrm{o}-\mathrm{i}} / T_{\mathrm{max}-\mathrm{i}}=1.0$ regardless of the backfill properties or slope inclination. The following should be pointed out: (a) the upper layer in the baseline problem has "tributary" zone of $0.75 \mathrm{~m}$ whereas most layers below have only $0.5 \mathrm{~m}$; (b) during construction, each layer will temporarily serve as an upper layer thus temporarily requiring the connection strength of the final upper layer; and (c) connection loads are affected by soil movement. The analysis used assumes that
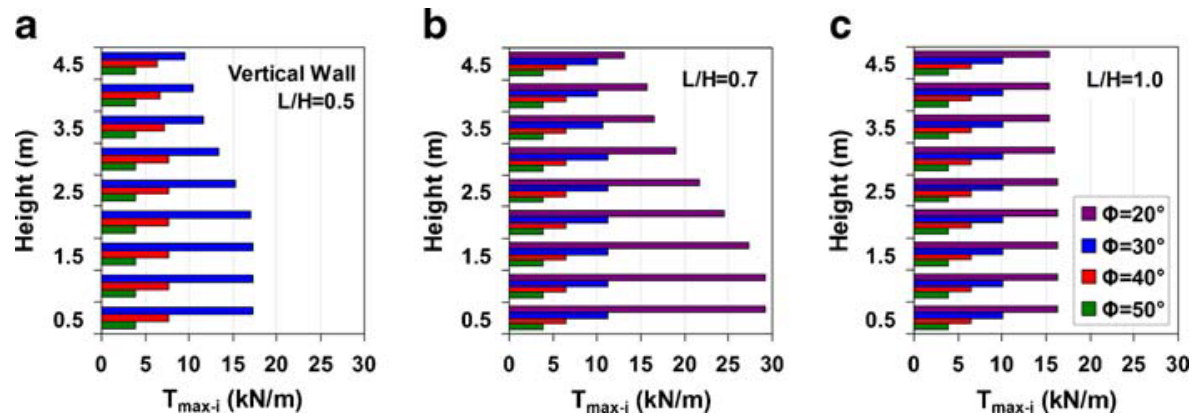

Fig. 20 Effects of length on $T_{\max -\mathrm{i} .}$ a $L / H=0.5$, b $L / H=0.7$, and $\mathbf{c} L / H=1.0$ 
a

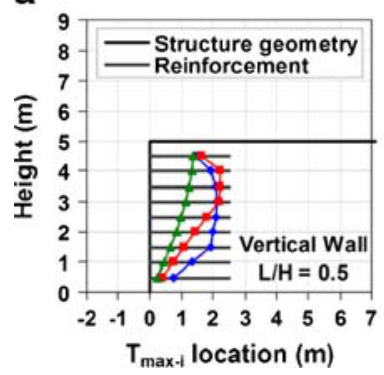

b

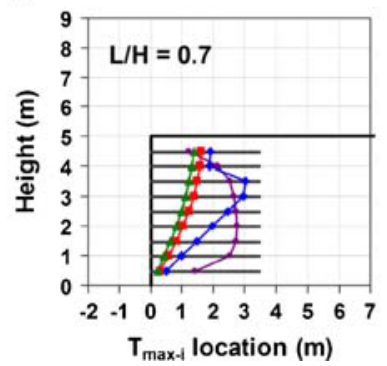

C

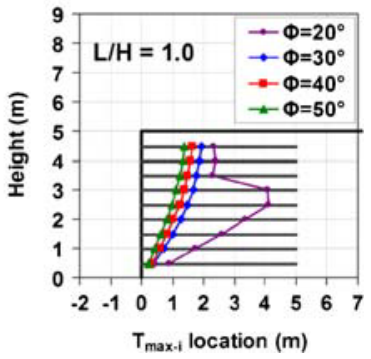

Fig. 21 Effects of length on locus of $T_{\text {max-i }}$. a $L / H=0.5, \mathbf{b} L / H=0.7$, and $\mathbf{c} L / H=1.0$

the soil can move to allow for an LE state; however, this may not always be the case especially at the connection. Discussion later shows that the connection loads have little effect on $T_{\max }$ as its influence is local; however, for some systems, such as those with mechanical connectors, it is prudent to use in design larger connection load than that rendered by the top-down analysis. In design, a constant value of $T_{\mathrm{o}-\mathrm{i}} / T_{\max -\mathrm{i}}=1.0$ will ensure little soil movements within the active wedge.

Figure $18 \mathrm{~b}$ indicates that for large $\phi$ values, the locus of $T_{\max -\mathrm{i}}$ is on a singular smooth curve extending from the toe to the crest. This curve is also a log spiral between the toe and crest. However, for low $\phi$, the curve ceases to be smooth signifying that for different layers $T_{\text {max-i }}$ is determined by different log spiral surfaces. In these cases, compound surfaces where the surfaces extend into the retained soil may become critical. Compound surfaces intersect less reinforcement layers thus potentially requiring more resistance for stability from the fewer intersected layers. This trend explains the required increase of $T_{\max -i}$ with depth for $\phi=20^{\circ}$ seen in Fig. 17a. Efficient utilization of reinforcement strength is when the locus is on a smooth curve, a potential slip surface, extending between the toe and crest. Hence, for a large $\phi$, the reinforcement strength is more uniformly mobilized thus becoming more efficient.

The tradeoff for more efficient use of reinforcement strength could come at the price of "excessively" long reinforcement. Refer to Fig. 19. It is clearly seen that for high values of $\phi$, the rear portion of lower layers is not being stressed; i.e., it is dormant. This apparent "waste" can be resolved by using there shorter reinforcement. However, from a practical perspective, such a layout is a potential source for costly construction error.

Figure 19 illustrated that front and rear resistive lengths can be significant near the crest, especially when low-quality backfill is used. Long pullout resistive lengths reduce the efficiency of the reinforcement as it limits the length over which its inherent strength can develop.

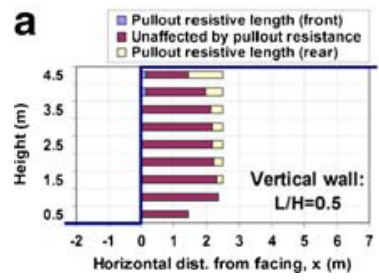

b

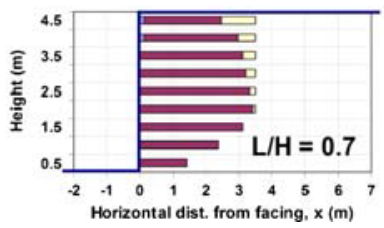

C

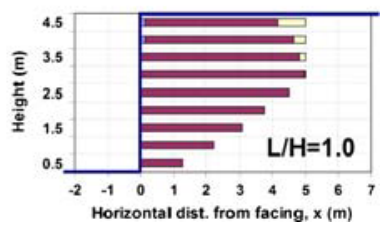

Fig. 22 Effects of length on pullout length $\left(\phi=30^{\circ}\right)$. a $L / H=0.5, \mathbf{b} L / H=0.7$, and $\mathbf{c} L / H=1.0$ 
a

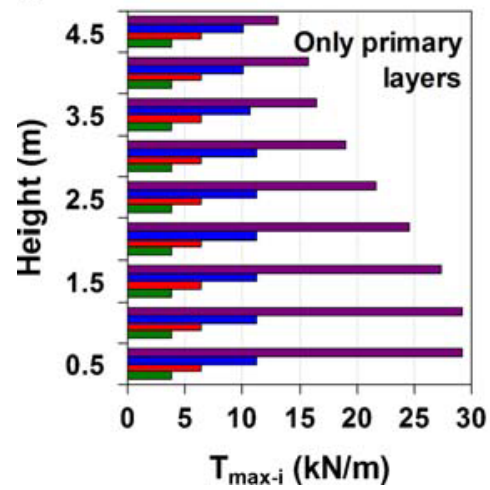

b

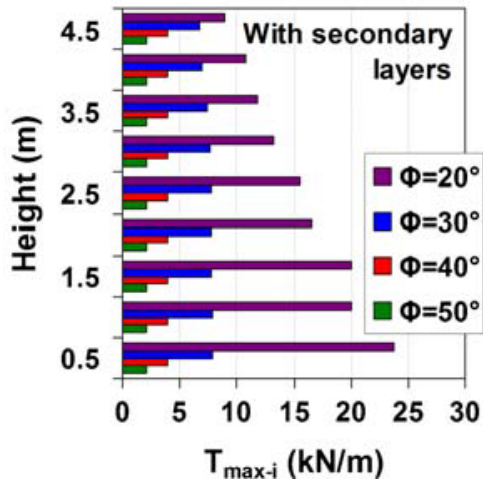

Fig. 23 Impact of 1.5-m long secondary layers on $T_{\text {max-i. }}$ a Without and $\mathbf{b}$ with

Figure 20 shows the impact of length of reinforcement. For $L / H=0.5, \phi=20^{\circ}$, and the spacing used, no stable structure is feasible. Simply, the pullout resistance capacity is insufficient to enable any reinforcement to maintain a stable system. However, for $\phi=50^{\circ}$, any length $L / H$ between 0.5 and 1.0 has no effect on $T_{\max -\mathrm{i}}$.

Figure 21 indicates $L / H=0.7$ is a borderline efficient length for a soil possessing $\phi$ slightly more than $30^{\circ}$. It also shows that for $L / H=0.5, \phi$ greater than $40^{\circ}$ would be most efficient.

Figure 22 is for $\phi=30^{\circ}$. It shows that the full length of short reinforcement (i.e., $L /$ $H=0.5$ ) is utilized except for the lowest layer. As the length increases, larger portions of lower layers are not mobilized, being dormant.

Figure 23 shows the impact of 1.5 -m long secondary layers, spaced every $0.5 \mathrm{~m}$, starting at $0.25 \mathrm{~m}$ above the toe elevation. As $\phi$ goes up, the effect of secondary layer on $T_{\text {max }-\mathrm{i}}$ is relatively more pronounced. Figure 24 provides an explanation for this phenomenon; the additional short layers add resistance intercepting slip surfaces, especially as $\phi$ goes up.

Secondary layers are sometimes used to enable better compaction next to the face. Its benefit in reducing $T_{\max -\mathrm{i}}$ is perhaps secondary and often is ignored. However, Leshchinsky [13] and Vulova and Leshchinsky [26] suggested that such layers can effectively reduce the connection load when using the conventional AASHTO's
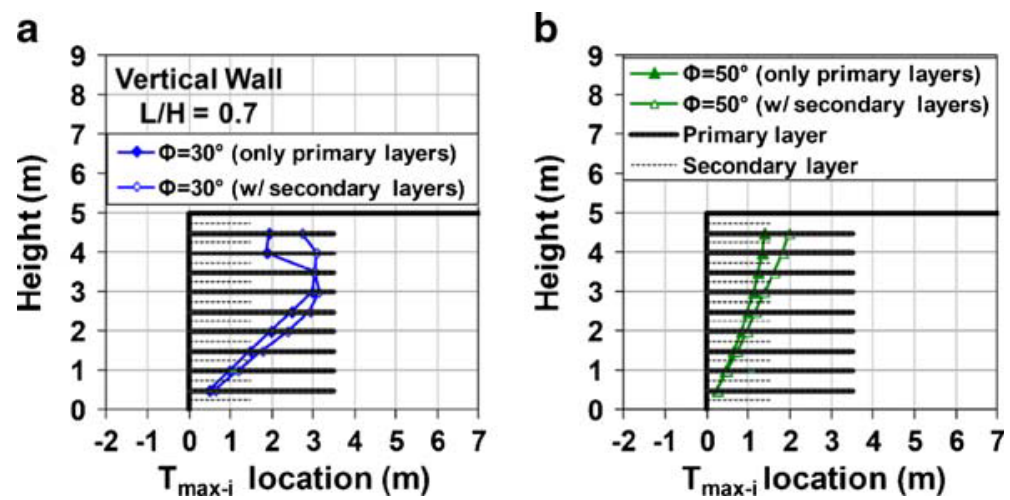

Fig. 24 Impact of 1.5-m Long secondary layers on locus of $T_{\max -\mathrm{i}}$. $\mathbf{a}$ Without and $\mathbf{b}$ with 
a

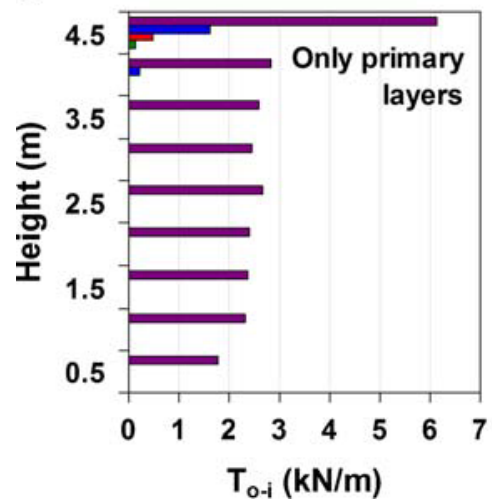

b

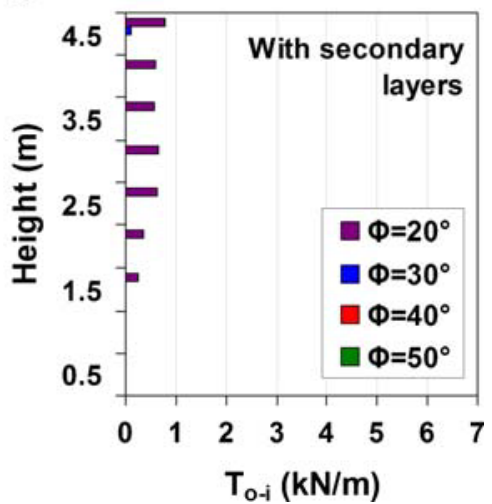

Fig. 25 Impact of 1.5-m long secondary layers on $T_{\mathrm{o}-\mathrm{i}}$. $\mathbf{a}$ Without and $\mathbf{b}$ with

analysis. In AASHTO's design reducing the required connection load could be significant for segmental block walls. Figure 25 shows that even in the current framework, the reduction in connection load is substantial in relative terms. This is evident when $\phi$ is low.

Figure 26 shows the impact of batter, $\omega$, on $T_{\max -\mathrm{i}}$. Increasing the batter dramatically reduces the required resistance of the reinforcement. For very small $\phi$ (say, 20 $0^{\circ} T_{\text {max-i }}$ at the bottom increases initially with slight increase in batter. The reason for that increase is due to compound failure becoming more critical thus decreasing the number of layers contributing to stability. Figure 27 shows the impact of batter on $T_{\mathrm{o}-\mathrm{i}} / T_{\max -\mathrm{i}}$. While $T_{\text {max-i }}$ goes down rapidly with increased batter, the ratio $T_{\mathrm{o}-\mathrm{i}} / T_{\max -\mathrm{i}}$ goes up with depth. While the connection strength required for zero batter was the greatest near the crest and vanishing with depth, for the inclined system, the required connection is not vanishing with depth. Figure 28 provides a rational explanation to this phenomenon. As seen, front end pullout resistive length increases at all depths. Simply, pullout is a function of overburden. The overburden decreases when considering points under the crest which turns into the slope surface when the batter goes up.

Figure 29 shows the locus of $T_{\max -i}$ as a function of batter. For lower $\phi$ values, increase in batter renders a more efficient system in a sense that the locus of $T_{\text {max-i }}$ extends along a single log spiral passing through all the layers.

a

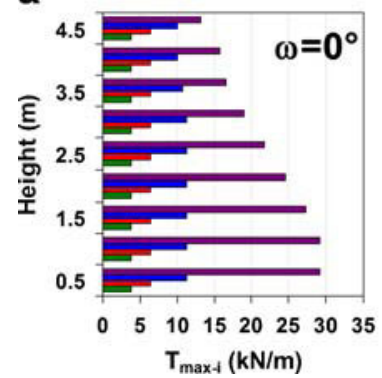

b

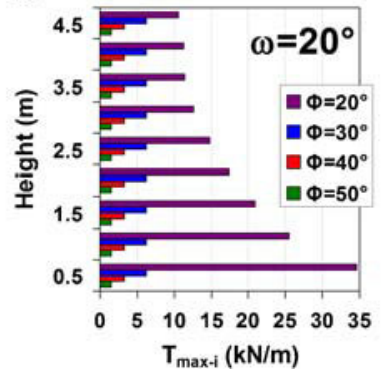

C

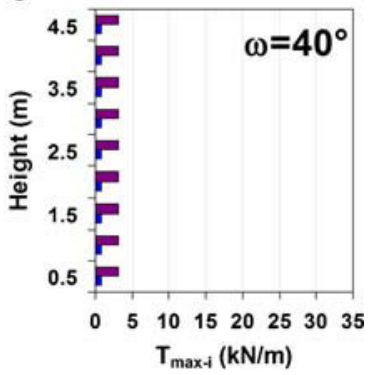

Fig. 26 Impact of batter on $T_{\max -\mathrm{i} .}$ a $\omega=0^{\circ}$, b $\omega=20^{\circ}$, and $\mathbf{c} \omega=40^{\circ}$ 

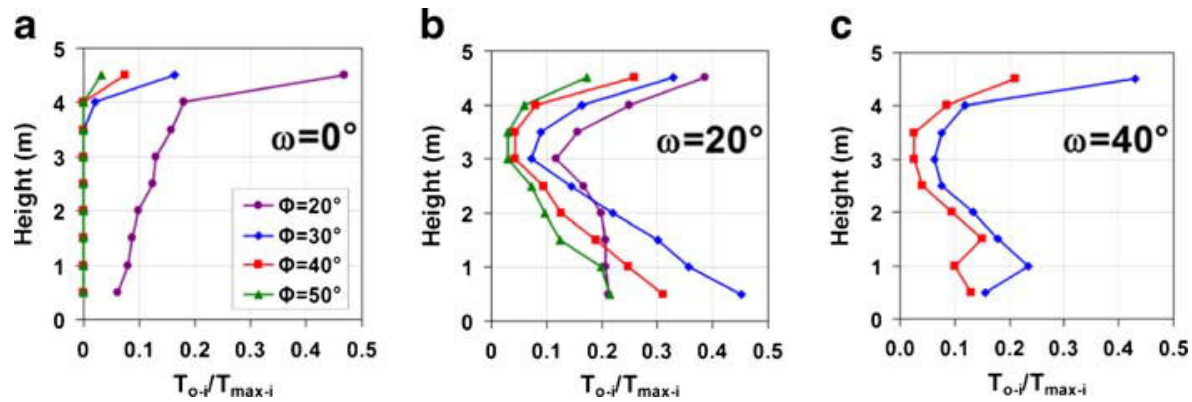

Fig. 27 Impact of batter on $T_{\mathrm{o}-\mathrm{i}} / T_{\text {max-i }} \cdot \mathbf{a} \omega=0^{\circ}, \mathbf{b} \omega=20^{\circ}$, and $\mathbf{c} \omega=40^{\circ}$

Figure 30 illustrates the effects of coverage ratio, $R_{\mathrm{c}}$. Not surprisingly, reducing its value from 100 to $75 \%$ results in an increase in $T_{\max -\mathrm{i}}$ as well as an increase in the required connection strength.

Figure 31 shows the impact of vertical spacing, $S_{\mathrm{v}}$, between reinforcement layers for $\phi=30^{\circ} . T_{\text {max-i }}$ and $T_{\mathrm{o}-\mathrm{i}}$ are decreasing nonlinearly with smaller spacing. With large $\phi$ values, this nonlinearity is less apparent.

Figure 32 implies that the impact of infinite backslope on $T_{\max -\mathrm{i}}$ is significant for low $\phi$. However, the impact diminishes when large friction angles are used. This is a consequence of a long backslope, essentially turning into an unstable slope when $\phi$ is low thus requiring a substantial structure for its support.

Figure 33 is a result of pseudostatic analysis. Simply, a horizontal component equals to $K_{\mathrm{h}} W$, where $K_{\mathrm{h}}$ is the horizontal seismic coefficient and $W$ is the weight of the soil mass defined by the analyzed log spiral, was introduced into the moment equilibrium equations when calculating the required resistance of reinforcement for stability. As can be seen, for $L / H=1.0$ and $\phi=30^{\circ}$, the load in the reinforcement increases significantly. The location of maximum load shifts backwards, especially in the lower layers which have large pullout resistance capacity. That is the dormant portions of the lower reinforcement are activated and mobilized during a seismic event. It is noted that for larger $\phi$, the increase in required tensile resistance is smaller and the shift of the maximum load backwards is also smaller. In seismic design, it is common to use a seismic coefficient that is half the peak ground acceleration, PGA (e.g., [23]); hence, Fig. 33 reflects a maximum PGA of $0.6 \mathrm{~g}$, a substantial magnitude.

To examine the impact of block facings, the simple model shown in Fig. 34 was used. Given the dimensions and bulk unit weight of the blocks, $\gamma_{\mathrm{u}}$, its effective weight,

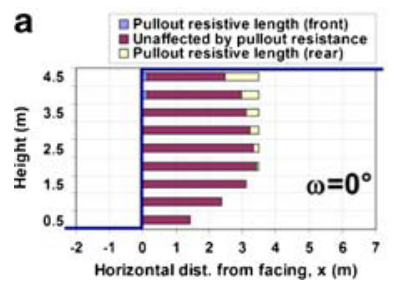

b

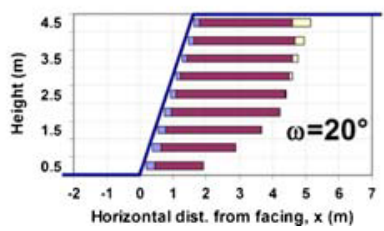

c

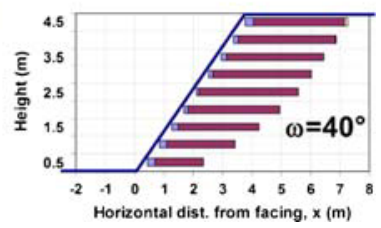

Fig. 28 Pullout resistive length for: a $\omega=0^{\circ}, \mathbf{b} \omega=20^{\circ}$, and $\mathbf{c} \omega=40^{\circ}$ 

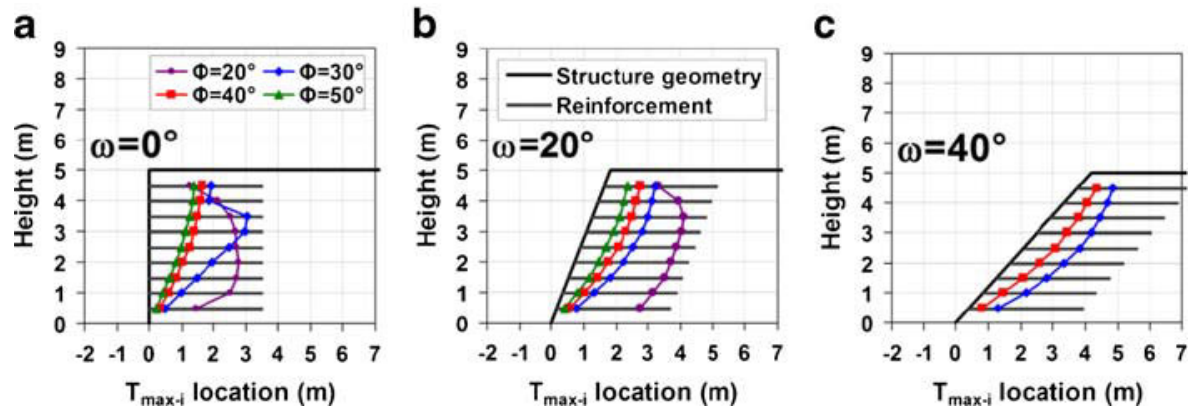

Fig. 29 Impact of batter on locus of $T_{\max -i}$

Fig. 30 Impact of coverage ratio, $R_{\mathrm{c}}$, for $\phi=30^{\circ}$
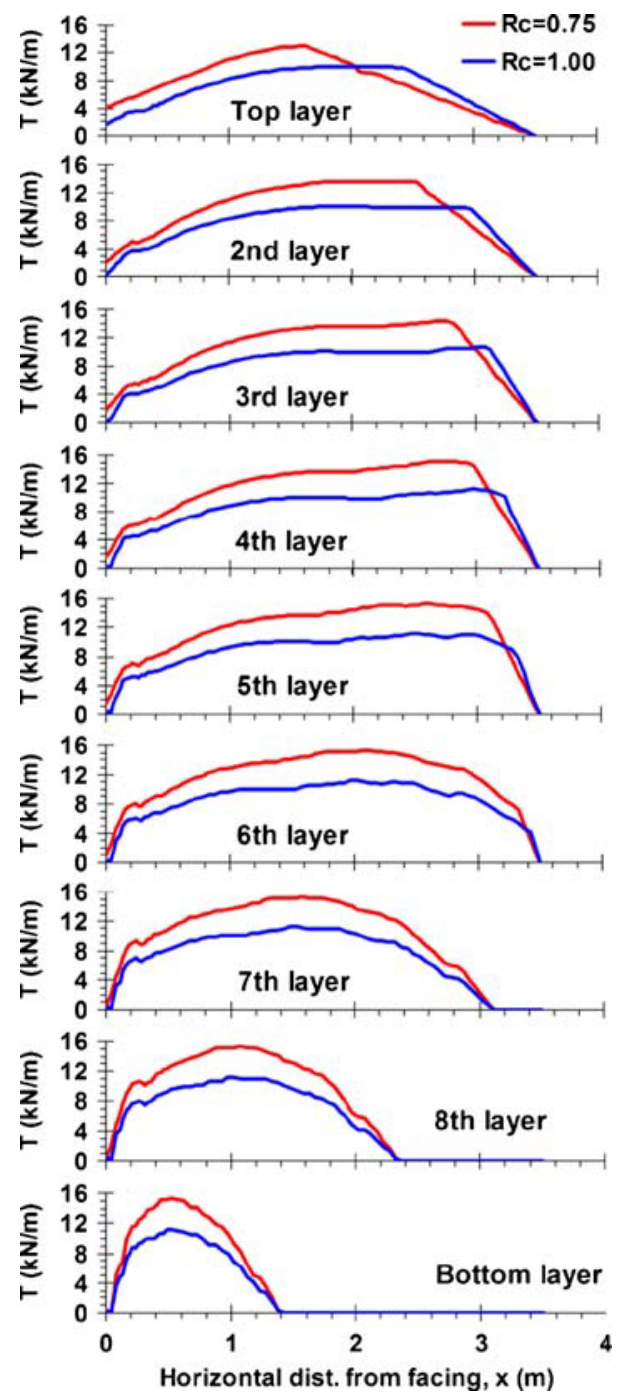
Fig. 31 Impact of reinforcement spacing, $S_{\mathrm{v}}$, for $\phi=30^{\circ}$
Spacing, Sv [m]:

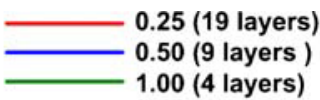

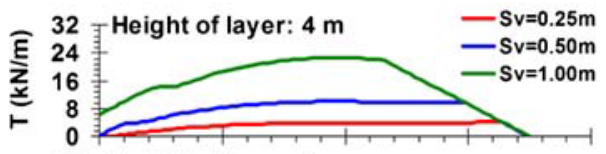
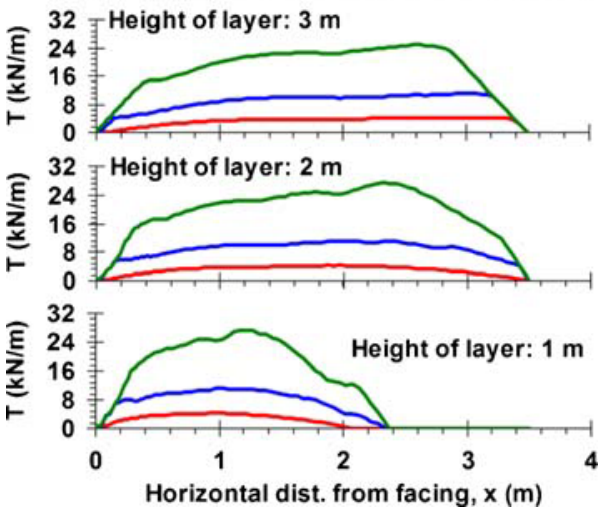

$R_{\mathrm{v}}$, based on effective block area, EA, above a desired elevation was calculated. Only the weight of facing above the interface at a desired elevation was calculated. This might be conservative but the simplicity of it is attractive for the purpose of this parametric study. Once $R_{\mathrm{v}}$ was calculated, the shear resistance value at that elevation, $R_{\mathrm{h}}$, could be assessed using a given value of interface friction. For block to block, this friction is denoted as $\delta_{\mathrm{b}-\mathrm{b}}$ and for block (or leveling pad) and foundation it is denoted by $\delta_{\mathrm{b}-\mathrm{f}}$. The modified moment equilibrium about the pole of the analyzed log spiral included the shear force $R_{\mathrm{h}}$ at the point the log spiral emerges. However, the vertical force $R_{\mathrm{v}}$ was not included as it was considered to be part of the weight of the test body (i.e., the facing was considered as part of the soil mass).

Figure 35 shows the distribution of the required tensile resistance along reinforcement layers. Changing $\delta_{\mathrm{b}-\mathrm{b}}$ from 0 to $50^{\circ}$ indicates that the reinforcement tension is
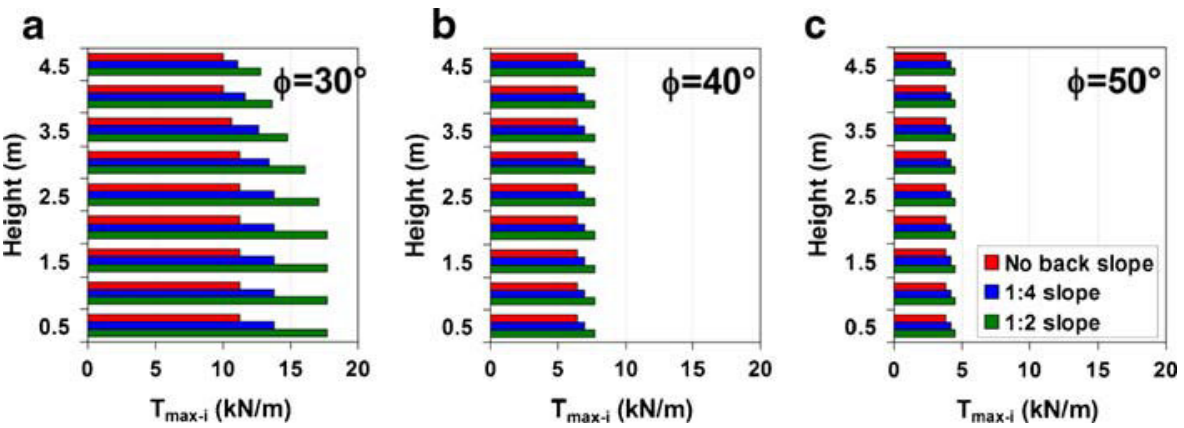

Fig. 32 Impact of Infinite backslope on $T_{\max -\mathrm{i}}$. a $\phi=30^{\circ}, \mathbf{b} \phi=40^{\circ}$, and $\mathbf{c} \phi=50^{\circ}$ 
Fig. 33 Impact of seismicity for $\phi=30^{\circ}$
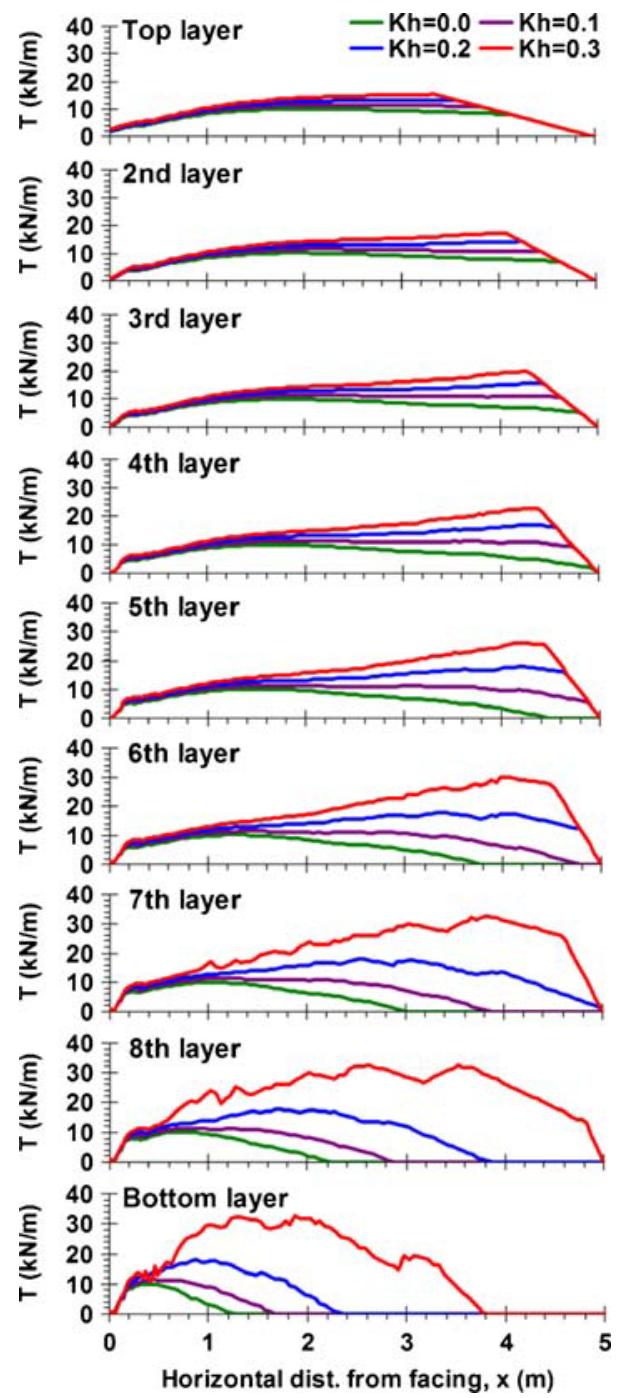

affected only at its front end. Increase in interblock friction decreases slightly the tensile load at the front end. In the present example it does not affect $T_{\max -\mathrm{i}}$. That is $\delta_{\mathrm{b}-\mathrm{b}}$ has local effect on the reinforcement, all in the vicinity of its connection to the block. However, $\delta_{\mathrm{b}-\mathrm{f}}$, the block to foundation shear resistance (i.e., toe resistance) has large impact on the required tensile resistance. Changing its $\delta_{\mathrm{b}-\mathrm{f}}$ value from 0 to $50^{\circ}$ decreases $\max \left(T_{\text {max-i }}\right)$ by over $50 \%$. This impactful toe resistance is produced by a typical $0.30-\mathrm{m}$ thick block. Also note that $T_{\max -\mathrm{i}}$ moves towards the face as the toe resistance increases. The large impact of toe resistance on $T_{\max }$ was discussed by Ehrlich et al. [8], Huang et al. [11], and Leshchinsky and Vahedifard [25]. 


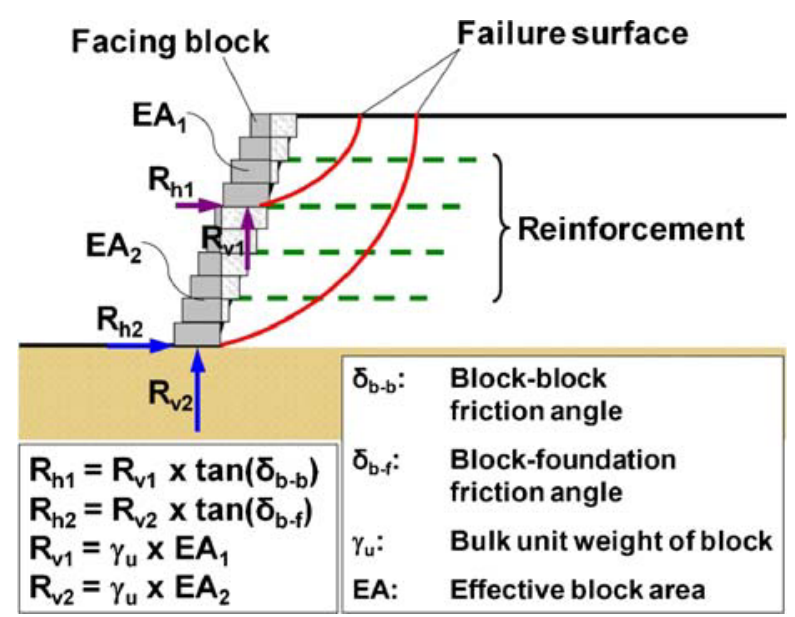

Fig. 34 Block-block and block-foundation shear resistance

Figure 36 shows that block-to-block friction has minor effect on $T_{\max -i}$ for low $\phi$ value; it has negligible effect for $\phi \geq 30^{\circ}$. However, toe resistance has large impact on $T_{\text {max-i }}$; this impact increases as $\phi$ increases.

Figure 37 shows the impact of toe resistance for flexible continuous facing. Such facing is simulated by block-to-block friction of $\delta_{\mathrm{b}-\mathrm{b}}=89.9^{\circ}$. Essentially, this interblock friction represents "infinite" shear strength between blocks thus preventing the formation of slip surface through the facing. However, the facing column is considered flexible thus allowing lateral soil movement to occur complying with the implicit analytical assumption that the soil can mobilize its strength. The already low required connection strength (see Fig. 35) then drops further. The peak $T_{\max -i}$ values remain nearly the same as in Fig. 35. That is flexible facing does not affect the required strength of the reinforcement; it may have a little effect on the connection load. This study indicates that the major impact of facing is its generated toe restraint or resistance. This resistance is usually ignored in design. Consequently it adds redundancy in the stability of the structure. Stated otherwise, not using block facing reduces the redundancy of the structure.

\section{Comparisons with Experimental Data}

The Norwegian Structure A 2(V):1(H) geogrid reinforced slope/wall, termed here as the Norwegian structure, was instrumented and tested by Fannin and Hermann [9]Fig. 38. As a facing, a wire grid was used. The reported moist unit weight was $\gamma=17$ $\mathrm{kN} / \mathrm{m}^{3}$ and the plane strain residual strength friction $\phi_{\mathrm{ps} \text {-residual }}=38^{\circ}$. Loads in the geogrid were measured using specially devised load cells.

Figure 39 shows the calculated tensile resistance along each primary reinforcement layer. It is calculated once for the reported residual shear strength of $38^{\circ}$ and once for an assumed peak shear strength value of $42^{\circ}$. It is noted that AASHTO recommends using 
Fig. 35 Impact of block-block and block-foundation shear resistance on required reinforcement tensile resistance distribution $\left(\phi=30^{\circ}\right.$; block thickness $\left.0.30 \mathrm{~m}\right)$
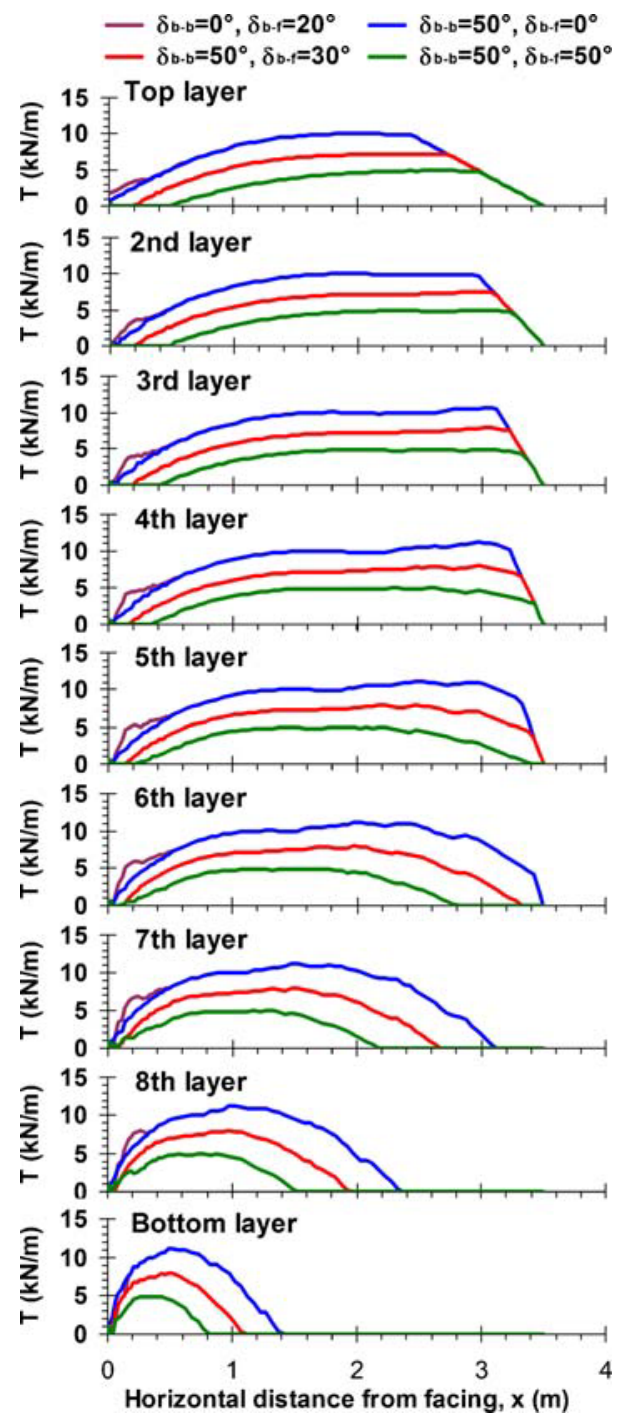

peak strength; hence, it makes sense choosing this value in the comparison here. The assumed peak strength of $42^{\circ}$ is reasonable based on common differences between peak and residual strength reported in the literature. Note the significant impact of $\phi$ on the required $T_{\max }$. As can be seen, when $\phi$ goes up, the locus of $T_{\max }$ becomes shallower, intercepting some secondary geogrid layers. Subsequently, $T_{\max }$ decreases as more layers contribute to stability along the critical surface. Figure 40 compares the measured and predicted $T_{\text {max-i }}$. The agreement is deemed good when peak soil strength is used. However, when residual value is considered, the framework produced twice the measured load. Note that the geogrid used by Fannin and Hermann [9] was stressed very little. It means that its deformation was minimal thus likely not allowing the soil to reach its residual strength. 
a

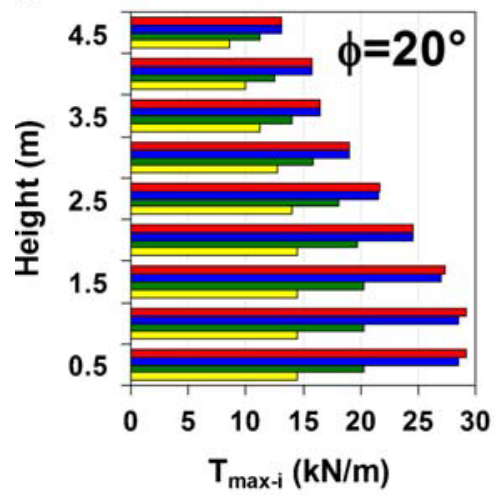

C

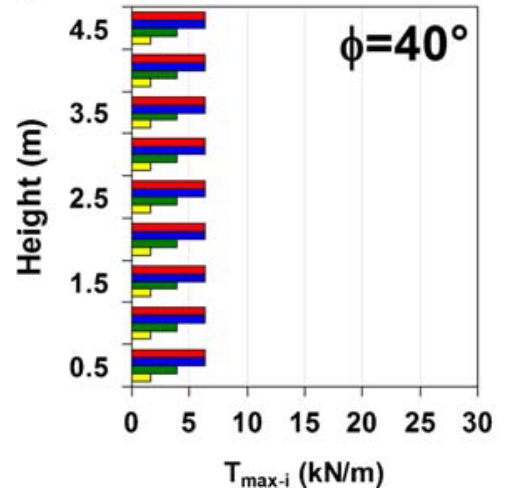

b

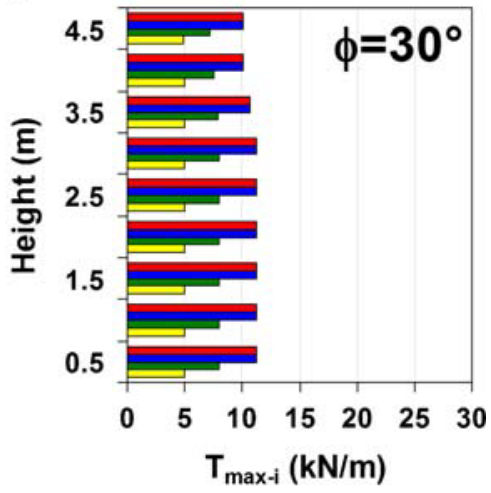

d

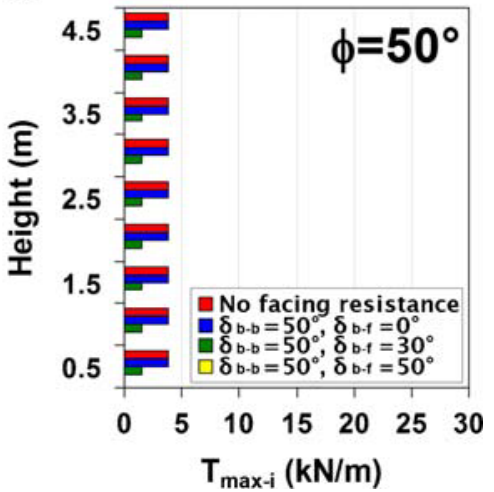

Fig. 36 Impact of block-block and block-foundation shear resistance on $T_{\max -i}$. a $\phi=20^{\circ}, \mathbf{b} \phi=30^{\circ}, \mathbf{c} \phi=40^{\circ}$, and $\mathbf{d} \phi=50^{\circ}$

The FHWA Wall (Algonquin) The section of FHWA wall was analyzed using data reported by Allen and Bathurst [3] - Fig. 41. This wall had relatively large facing blocks (0.6-m thick). The parametric studies in the previous section indicate that toe resistance could be substantial. No measured data regarding this resistance was reported. Hence, three values of interface friction between the leveling pad and the foundation soil, $\delta_{\mathrm{b}-\mathrm{f}}$, were assumed: $0^{\circ}, 30^{\circ}$, and $43^{\circ}$. Figure $42 \mathrm{a}$ shows that for the case of horizontal crest, the measured $T_{\text {max-i }}$ reasonably corresponded to $\delta_{\mathrm{b}-\mathrm{f}}$ between $30^{\circ}$ and $43^{\circ}$ although with some scatter. Figure $42 \mathrm{~b}$ shows $T_{\max -\mathrm{i}}$ for the backslope surcharge. Similar to the case of the horizontal crest, the measured $T_{\text {max-i }}$ reasonably corresponded to $\delta_{\mathrm{b}-\mathrm{f}}$ between $30^{\circ}$ and $43^{\circ}$. Accurate comparisons in this case history are not warranted as the available data about toe resistance can only be speculated.

Centrifuge Geotextile-Reinforced Structure Zornberg et al. [28] report the results of centrifugal tests on dry sand that was placed by pluviation (i.e., there was no apparent cohesion due to moisture). They used properly modeled geotextile, spinning the model to an acceleration at which collapse occurred (Fig. 43). The tested slope/wall had 
Fig. 37 Tensile resistance distribution for continuous flexible facing $\left(\phi=30^{\circ}\right)$

$-\delta b-b=89.9^{\circ}, \delta b-f=0^{\circ}$

$-\delta b-b=89.9^{\circ}, \delta_{b-f}=30^{\circ}$

$-\delta b-b=89.9^{\circ}, \delta_{b-f}=50^{\circ}$
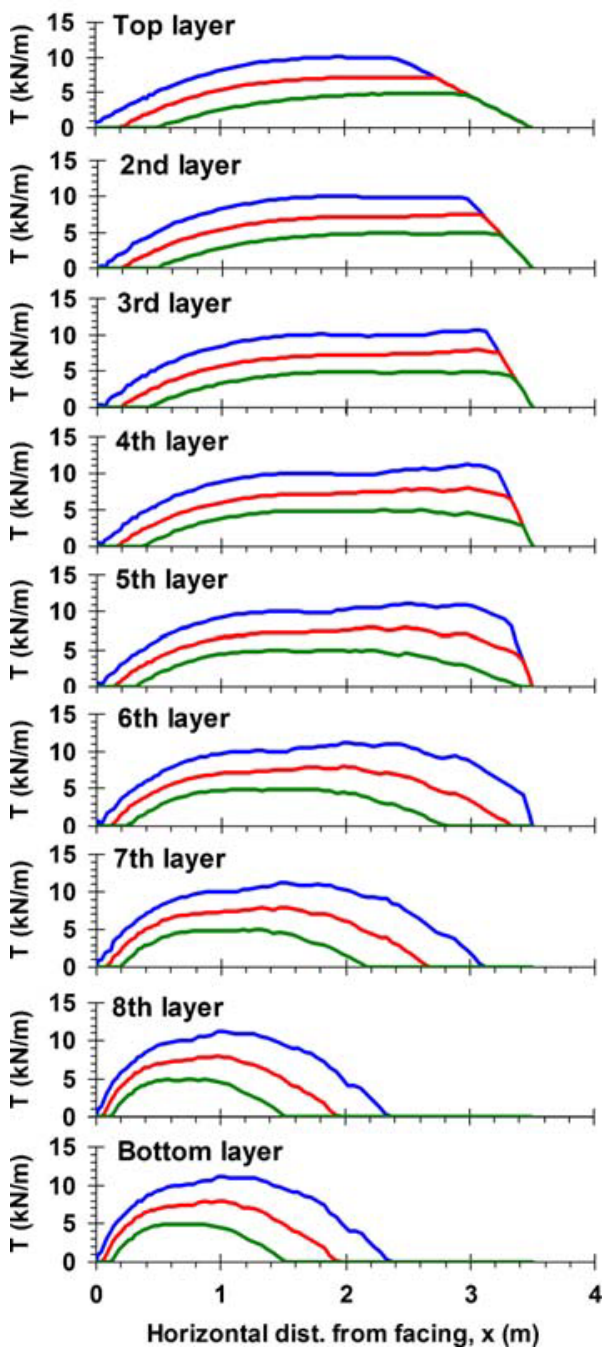

wrapped face; the re-embedded tails of the geotextile can be considered as secondary layers.

Figure 44 shows the general layout of reinforcement in the model tested by Zornberg et al. [28]. Figure 45 shows the dimensions of the prototype corresponding to the centrifugal model at failure. It also shows the locus of predicted and measured location of $T_{\text {max-i }}$. Since the exact locus of $T_{\text {max }-i}$ is rather insensitive in the LE calculations, the agreement is considered good. The calculated $T_{\text {max-i }}$ in the prototype was $9.54 \mathrm{kN} / \mathrm{m}$; the ultimate strength of the geotextile in the prototype as reported by Zornberg et al. 


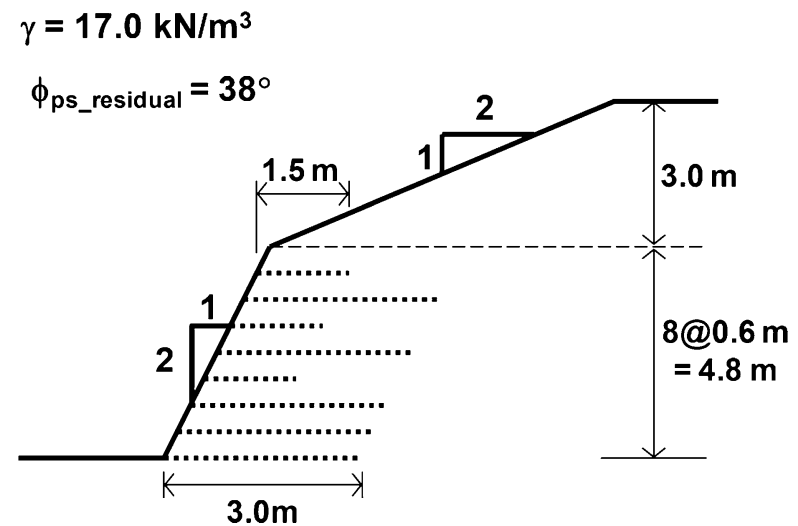

Fig. 38 The Norwegian wall: geometry and geogrid layout (after [9])

[28] was $9.66 \mathrm{kN} / \mathrm{m}$. Clearly, the calculated locus of $T_{\max -\mathrm{i}}$ and the actual values $T_{\max -\mathrm{i}}$, both relevant to design while also signifying the essence of the presented framework, are in as good of an agreement as one can expect.

\section{Concluding Remarks}

Presented is a LE-based framework producing data required for ultimate limit state (ULS) design. To assess the reasonableness of the proposed framework, extensive parametric studies were conducted. Other LE methods can be implemented in the framework thus making it capable of dealing with more complex problems. It is noted that illustrative examples using the presented framework are provided by Kang et al. [12].

The following conclusions can be drawn from the parametric studies:

a. Tensile load in the reinforcement may depend on its length. This is contrary to some current design approaches (e.g., AASHTO) where length is not part of the formulation in determining this load.
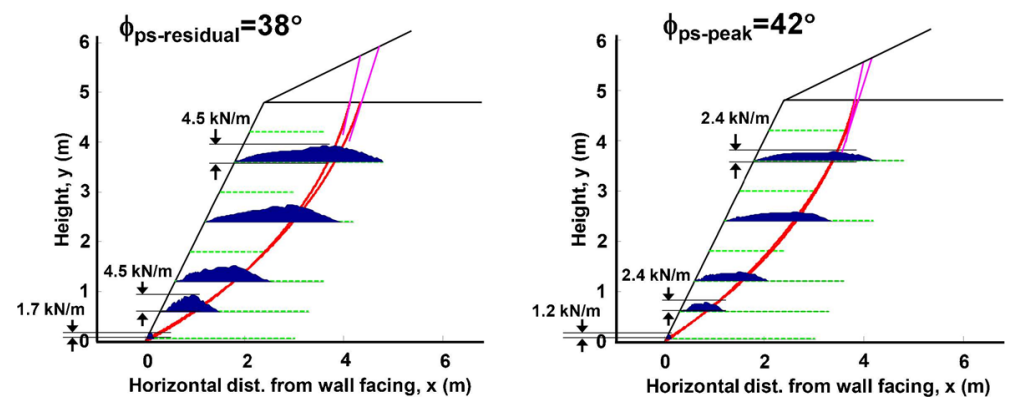

Fig. 39 The Norwegian wall: calculated tension along primary geogrid layers 
a

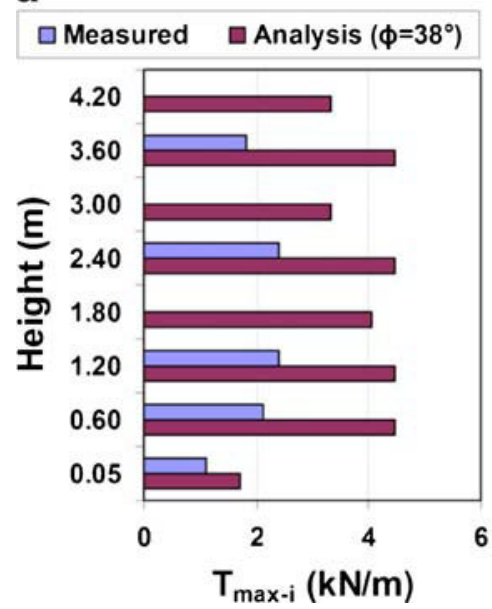

b

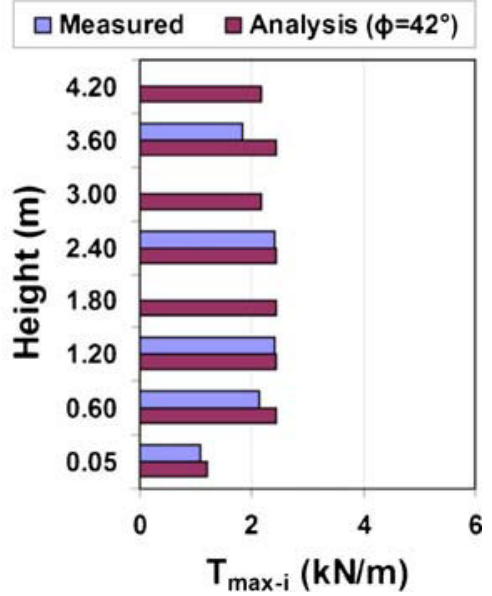

Fig. 40 The Norwegian wall: measured and predicted loads for: a residual $\phi$ and $\mathbf{b}$ peak $\phi$

b. Overly long reinforcement could be dormant (unused) along its rear end. However, "overly long" depends on the soil strength, $\phi$. For large $\phi$ values, $L /$ $H=0.7$ renders much of the rear portion of the reinforcement in lower elevations unused under static loading. Under seismic loading, this dormant portion might be needed for stability.

c. For typical walls, $\max \left(T_{\max }\right)$ can be about half the value produced by some current design approaches (e.g., AASHTO). This means that reinforcement with possibly half strength can be used. The exact reduction in required strength depends on the specific problem.

d. Connection load significantly increases with low percent coverage and/or lowquality fill and/or large spacing.

e. The presented methodology to find connection loads is contrary to the arbitrary value of $T_{\mathrm{o}}=T_{\max }$. Typically, the connection load is less than $0.5 T_{\max }$. For vertical walls, the connection load at lower elevations is very small provided that the soil

Fig. 41 Section of FHWA wall (reproduced after [3])

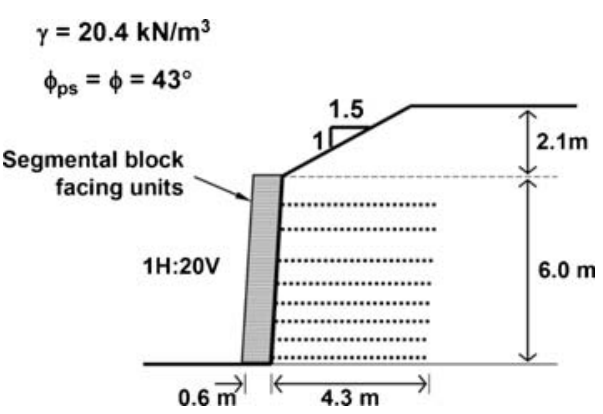


a

$$
\begin{aligned}
& \square \phi=43^{\circ}, \delta b-f=0 \\
& \square \phi=43^{\circ}, \delta b-f=30^{\circ} \\
& \square \phi=43^{\circ}, \delta b-f=43^{\circ}
\end{aligned}
$$

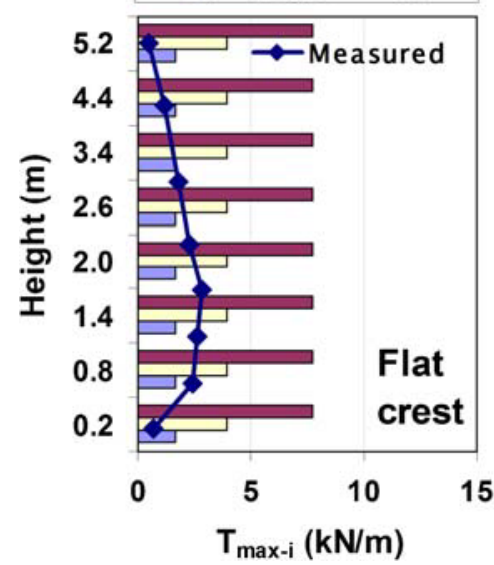

b

\section{Backslope \\ ("surcharged")}

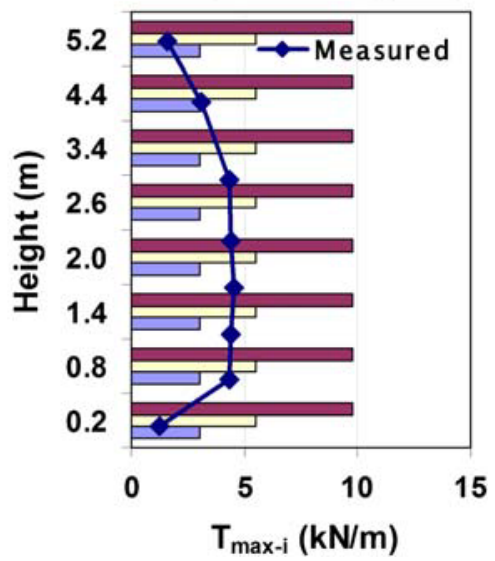

Fig. 42 Measured and predicted loads in FHWA wall: a horizontal crest and $\mathbf{b}$ backsloped crest

can move sufficiently to mobilize its strength. However, as discussed at the end linking the results to design, connection strength might be needed for performance related to deformations.

f. $T_{\mathrm{o}} / T_{\max }$ may increase with depth for large batter since the front end pullout decreases due to lower overburden pressure. However, the ratio is much less than AASHTO's value of 1.0. In absolute terms, $T_{\mathrm{o}}$ gets smaller as the batter goes up while the ratio $T_{\mathrm{o}} / T_{\max }$ may increase as $T_{\max }$ decreases at a different rate than $T_{\mathrm{o}}$.

g. Secondary or intermediate reinforcement significantly reduces $T_{\mathrm{o}}$ and $T_{\max }$. Its impact on $T_{\mathrm{o}}$ and/or $T_{\max }$ depends on the length of these secondary layers.

h. In slope stability analysis, Fs is applied to the soil strength, $\phi$. In reinforced soil structures the designer can select $\phi$ (as currently done in AASHTO), a value with which the designer feels comfortable and then apply a factor on the

Fig. 43 Failed geotextilereinforced slope/wall in centrifuge modeling (courtesy Prof. Zornberg)

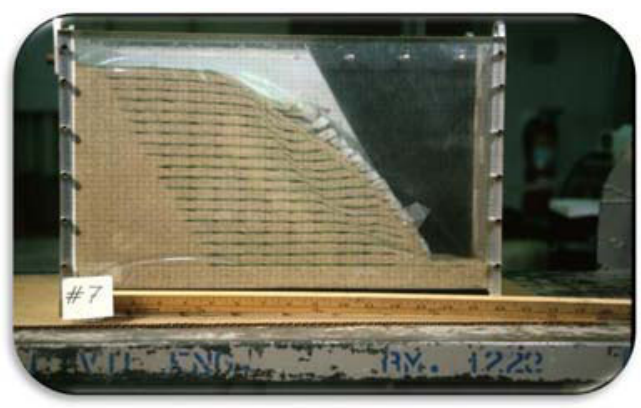


Fig. 44 General layout and dimensions of tested centrifugal models (after [28])

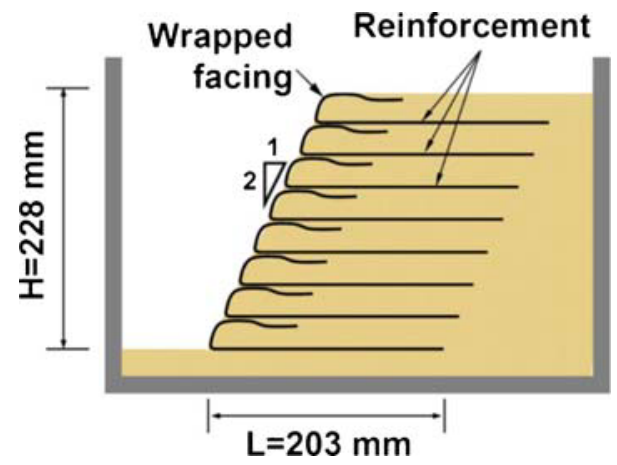

reinforcement. This will yield shorter and weaker reinforcement than applying Fs on the soil strength. The alternative is imposing "double taxation" on the reinforcement; once through using a reduced $\phi$ by Fs which results in inflated reinforcement strength and length and second directly increasing by a factor the calculated $T_{\max }$.

i. The small connection load, typically $T_{\mathrm{o}} / T_{\max } \leq 0.5$, implies sustained load at the connection that is lower than the long-term design strength value. Hence, if creep rupture is used in determining the required strength of reinforcement, the applied creep reduction factor (e.g., AASHTO) at the connection is excessive.

j. A few comparisons with experimental work show the presented approach to yield reasonable results.

k. Extending the framework to deal with complex problems follows well-established practice:

i. Consider complex geometries without the artificial distinction between wall and slope

ii. Consider water

iii. Consider nonuniform soil strata (e.g., gravel at the lower wall for tidal changes and finer material above)

iv. Consider various surcharge loads

v. Consider poor foundation soil
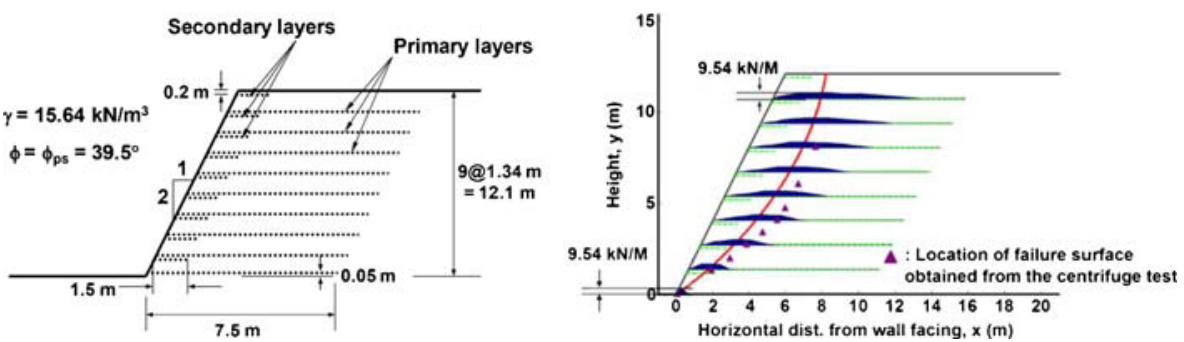

Fig. 45 Prototype at failure corresponding to centrifugal model (left) and predicted and measured locus of $T_{\max -\mathrm{i}}($ right $)$ 
Fig. 46 The link between results from framework and specification for design

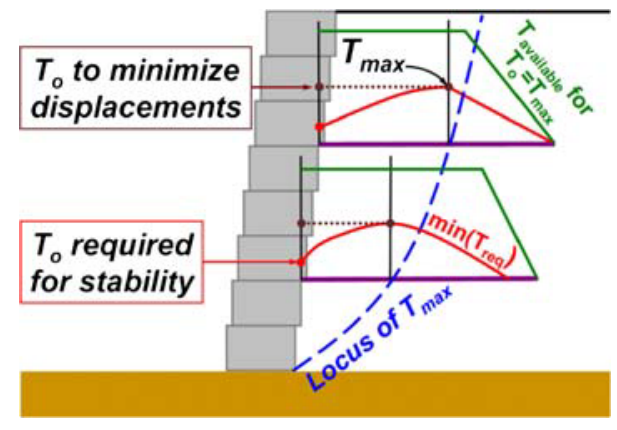

1. The framework can be straightforward integrated with any acceptable slope stability analysis using LE. Hence, unlike the current approach, the framework does not require a synergistic approach to deal with internal/external stability. It all can be done consistently using one tool.

m. The framework emanates from basic statics where free body (limit) equilibrium is sought at any point along any reinforcement layer. This simple concept yields an ULS solution that addresses many relevant design issues.

n. The safety map approach is an efficient optimization approach if the alternative, less explicit, conventional LE analysis is used.

Refer to Fig. 46. It shows the link between the calculated results using the framework and the strength values that should be specified in design marked as $T_{\text {available. It }}$ can be seen that along most of the length of the reinforcement the safety ratio exceeds the minimum required ratio of $T_{\text {available }} / T_{\text {max }}$. This is a redundancy stemming from the fact that $T_{\text {req }}(x)$ is not constant along $x$. The analysis in this work shows that the need for surficial stability (i.e., stability in the zone of the facing) does not require much resistance or significant connection strength. However, Fig. 46 implies that while the calculated $T_{\mathrm{o}}$ is rather small, the required value in design imposes $T_{\mathrm{o}}=T_{\max }$ meaning that $T_{\text {available }}$ needs to be available also at the connection. The reason for such a requirement is obviously not related to stability. It is related to construability and the behavior of the active wedge. Good connection enables good compaction near the facing, an element important for structural performance. Furthermore, it keeps the active wedge in a true coherent mass state thus minimizing deformations within that zone. Soil confining stresses are kept high thus producing stiff mass and ensuring 'local' stability by preventing possible progressive failure. Again, it ensures good structural performance.

Finally, under "normal" field conditions it is likely that apparent cohesion and toe resistance exist. These elements are ignored in design. Ultimate limit state (ULS) refers to extreme condition needed over short time relative to the lifespan of structure. Consequently, long-term sustained load could be much smaller than needed for ULS, probably half the value. In such a case, smaller creep reduction factor can be used as the duration under which the load associated with ULS is much shorter than the lifespan of the reinforced structure. However, this observation needs further verification. 


\section{References}

1. AASHTO: Standard specifications for highway bridges, 17th edn. AASHTO, Washington, DC (2002)

2. AASHTO: LRFD bridge design specifications, 4th edn. AASHTO, Washington, DC (2007)

3. Allen, T.M., Bathurst, R.J.: "Prediction of soil reinforcement loads in mechanically stabilized earth (MSE) walls.” Rep. No. WARD-522.1. Washington State Department of Transportation, Seattle (2001)

4. Baker, R., Klein, Y.: An integrated limiting equilibrium approach for design of reinforced soil retaining structures: part I-formulation. Geotext Geomembr 22(3), 119-150 (2004)

5. Baker, R., Klein, Y.: An integrated limiting equilibrium approach for design of reinforced soil retaining structures: part II — design examples. Geotext Geomembr 22(3), 151-177 (2004)

6. Baker, R., Leshchinsky, D.: Spatial distributions of safety factors. J. Geotech Geoenviron Eng 127(2), 135-145 (2001)

7. Bathurst, R.J., Walters, D., Vlachopoulos, N., Burgess, P., Allen, T.M.: "Full scale testing of geosynthetic reinforced walls". ASCE special publication. Proceedings of GeoDenver 2000, Denver (2000)

8. Ehrlich, M., and Becker, L. D. B., "Reinforced soil wall measurements and predictions." Proc., 9th Int. Conf. on Geosynthetics: Geosynthetics for a challenging world, E. M. Palmeira, D. M. Vidal, A. S. J. F. Sayao, and M. Ehrlich, eds., Vol. 1, IGS Brasil and ABMS, Brazil, 2010, 547-559.

9. Fannin, R.J., Hermann, S.: Performance data for a sloped reinforced soil wall. Can Geotech J 27(5), 676686 (1990)

10. Han, J., Leshchinsky, D.: General analytical framework for design of flexible reinforced earth structures. J Geotech Geoenviron Eng 132(11), 1427-1435 (2006)

11. Huang, B., Bathurst, R.J., Hatami, K., Allen, T.M.: Influence of toe restraint on reinforced soil segmental walls. Can Geotech J 47(8), 885-904 (2010)

12. Kang, B., "Framework for design of geosynthetic reinforced segmental retaining walls.", $\mathrm{PhD}$ dissertation, Univ. of Delaware, (2013)

13. Leshchinsky, D.: Alleviating connection load. Geotech Fabr Rep 18(8), 34-39 (2000)

14. Leshchinsky, D.: On global equilibrium in design of geosynthetic reinforced walls. J Geotech Geoenviron Eng ASCE 135(3), 309-315 (2009)

15. Leshchinsky, D., "Geotextile reinforced earth: numerical procedure," Research Report CE84 45 , Department of Civil Engineering, Univ. of Delaware, (1984)

16. Leshchinsky, D., "Design procedure for geosynthetic reinforced steep slopes," Geotechnical Laboratory, US Army Corps of Eng., Waterways Experiment Station, Report REMR-GT-23, (1997), Vicksburg, Mississippi

17. Leshchinsky, D., "Issues in geosynthetic-reinforced soil." Keynote paper, Proc., Int. Symposium on Earth Reinforcement Practice, Vol. 2, Kyushu, Japan, Balkema, Rotterdam, The Netherlands, 871-897 (1992)

18. Leshchinsky, D., "The power of software in reinforcement applications: Part I, Part II, Part III, Part IV," Geotechnical Fabrics Report, Vol. 23, 2005,Numbers 5, 6, 7, 8

19. Leshchinsky, D., "Topics in geosynthetic reinforced earth structures," Keynote Paper, Proc., Conferenze Di Geotechnica Di Torino, XXIII Cicloe: Earth Retaining Structures and Slope Stabilization: Theory, Design, and Application, (2011)

20. Leshchinsky, D., Han, J.: Geosynthetic reinforced multitiered walls. J Geotech Geoenviron Eng 130(12), 1225-1235 (2004)

21. Leshchinsky, D., Lambert, G.: Failure of cohesionless model slopes reinforced with flexible and extensible inclusions. Transp Res Rec 1330, 54-63 (1991)

22. Leshchinsky, D., Ling, H.I., Hanks, G.: Unified design approach to geosynthetic reinforced slopes and segmental walls. Geosynth Int 2(4), 845-881 (1995)

23. Leshchinsky, D., Ling, H.I., Wang, J.-P., Rosen, A., Mohri, Y.: Equivalent seismic coefficient in geocell retention systems. Geotext Geomembr 27(1), 9-18 (2009)

24. Leshchinsky, D., Tatsuoka, F.: Geosynthetic reinforced walls in the public sector: performance, design, and redundancy. Geosynth Mag (formerly GFR) 31(3), 12-21 (2013)

25. Leshchinsky, D., Vahedifard, F.: Impact of Toe resistance in reinforced masonry block walls: design dilemma. J Geotech Geoenviron Eng ASCE 138(2), 236-240 (2012)

26. Vulova, C. and., Leshchinsky, D., "Effects of secondary reinforcement layers on the behavior of block walls," Proceedings of the 7th International Conference on Geosynthetics, Nice, France, Vol. 1, 321-324 (2002), eds: Delmas, Gourc and Girard. Balkema

27. Washington State Dept. of Transportation (WSDOT), Geotechnical design manual, Chapter 15, Olympia, WA, (2010)

28. Zornberg, J.G., Sitar, N., Mitchell, J.K.: Limit equilibrium as basis for design of geosynthetic reinforced slopes. J Geotech Geoenviron Eng 124(8), 684-698 (1998) 